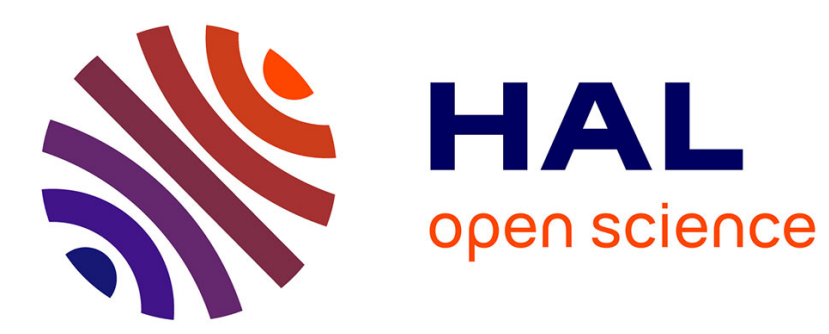

\title{
Translation of harmonic spinors and interacting weyl fermions on homogeneous spaces
}

\author{
Salah Mehdi, Nicolas Prudhon
}

\section{To cite this version:}

Salah Mehdi, Nicolas Prudhon. Translation of harmonic spinors and interacting weyl fermions on homogeneous spaces. Salah Mehdi; Nicolas Prudhon. TJC 2015: Geometric and Harmonic Analysis on Homogeneous Spaces and Applications, 207, Springer, pp.185-208, 2018, Springer Proceedings in Mathematics \& Statistics (PROMS), 978-3-319-65180-4. 10.1007/978-3-319-65181-1_7 . hal03336288

\section{HAL Id: hal-03336288 \\ https://hal.science/hal-03336288}

Submitted on 7 Sep 2021

HAL is a multi-disciplinary open access archive for the deposit and dissemination of scientific research documents, whether they are published or not. The documents may come from teaching and research institutions in France or abroad, or from public or private research centers.
L'archive ouverte pluridisciplinaire HAL, est destinée au dépôt et à la diffusion de documents scientifiques de niveau recherche, publiés ou non, émanant des établissements d'enseignement et de recherche français ou étrangers, des laboratoires publics ou privés. 


\title{
TRANSLATION OF HARMONIC SPINORS AND INTERACTING WEYL FERMIONS ON HOMOGENEOUS SPACES
}

\author{
S. MEHDI AND N. PRUDHON
}

\begin{abstract}
We show that the image of the Poisson map, defined by Mehdi and Zierau in [MZ14b], which intertwines principal series representations with a submodule of the kernel of the cubic Dirac operator, commutes with the translation functor. As a byproduct, we obtain a systematic geometric process which produces interacting Weyl fermions with a fixed energy level on homogeneous spacetimes.
\end{abstract}

\section{In honor of Professor Jean Ludwig}

\section{INTRODUCTION}

The relativistic quantum dynamics of a free massive spin- $\frac{1}{2}$ particle on the Minkowski spacetime is described by the Dirac equation (1928):

$$
\left[\gamma_{0} \frac{1}{c} \frac{\partial}{\partial t}+\sum_{j=1}^{3} \gamma_{j} \frac{\partial}{\partial x_{j}}\right] \psi=\frac{m c}{\imath \hbar} \psi,
$$

where $c$ is the velocity of light in vacuum, $m$ is the mass at rest and the $\gamma_{j}$ 's are $4 \times 4$ complex matrices (built from Pauli matrices) satisfying the following conditions:

$$
\gamma_{0}^{2}=I, \gamma_{j}^{2}=-I \text { for } j=1,2,3 \text {, and } \gamma_{j} \gamma_{k}+\gamma_{k} \gamma_{j}=0 \text { for all } j \neq k .
$$

Solutions to Dirac equation are called Dirac fermions (e.g., electrons, protons, neutrons).

If we choose units such that both the velocity of light and the Planck constant equal 1 , then the Dirac operator $\widehat{\mathcal{D}}$ is the matrix-valued differential operator defined by:

$$
\widehat{\mathcal{D}}=\gamma_{0} \frac{\partial}{\partial t}+\sum_{j=1}^{3} \gamma_{j} \frac{\partial}{\partial x_{j}} .
$$

It is a first order Lorentz-invariant differential operator acting on sections of the spin bundle $\mathcal{S}$ over Minkowski spacetime. The spin bundle splits into half-spin bundles $\mathcal{S}^{+}$and $\mathcal{S}^{-}$so that

Key words and phrases. Dirac operator; harmonic spinor; homogeneous space; admissible representation; translation functor; Weyl fermion, interaction.

2000 Mathematics Subject Classification. Primary 22E46; Secondary 22F30; 22E70. 
$\widehat{\mathcal{D}}^{ \pm}:=\left.\widehat{\mathcal{D}}\right|_{\mathcal{S} \pm}$ sends sections of $\mathcal{S}^{ \pm}$to sections of $\mathcal{S}^{\mp}$. Hence the Dirac equation for massless particles reads as the Weyl equations:

$$
\widehat{\mathcal{D}}^{ \pm} \psi=0
$$

whose solutions are the (massless) Weyl fermions (e.g., neutrinos, antineutrinos). In particular, given the splitting $\mathcal{S}=\mathcal{S}^{+}+\mathcal{S}^{-}$of the spin bundle, Dirac fermions can be treated as a combination of Weyl fermions. While Hermann Weyl predicted the existence of such particles in the late 1920's, it was only on July 2015 that a research team from Princeton University reported the first observation of Weyl fermions (http://phys.org/news/2015-12-weyl-fermiondiscovery-ten-breakthrough.html).

In this paper, we are interested in interactions with Weyl fermions. More precisely, attached with a finite-dimensional representation $E$ of $\operatorname{Spin}(1,3)$, there are a bundle $\mathcal{E}$ over Minkowski spacetime and a twisted Dirac operator $\widehat{\mathcal{D}}^{ \pm}(E)$ defined by:

$$
\widehat{\mathcal{D}}^{ \pm}(E)=\left(\gamma_{0} \otimes I_{\mathcal{E}}\right) \frac{\partial}{\partial t}+\sum_{j=1}^{3}\left(\gamma_{j} \otimes I_{\mathcal{E}}\right) \frac{\partial}{\partial x_{j}}
$$

which acts on sections of the bundle $\mathcal{S}^{ \pm} \otimes \mathcal{E}$, where $I_{\mathcal{E}}$ is the identity operator on $\mathcal{E}$. The field equations for the Weyl fermions, interacting with particles 'living' in the bundle $\mathcal{E}$, are then the following twisted Weyl equations:

$$
\widehat{\mathcal{D}}^{ \pm}(E) \psi=0
$$

Solutions are composite objects, resulting from interactions of the Weyl fermions with particles 'living' in $\mathcal{E}$. We shall provide a geometric process which produces interacting Weyl fermions with a fixed energy level on general homogeneous spacetimes. Our approach is based on representation theory of semisimple Lie groups.

More precisely, suppose $G / H$ is a homogeneous space where $G$ is a connected non-compact semisimple Lie group with finite center and $H$ is a closed connected reductive subgroup of $G$. Suppose $S$ is the spin representation of $H$ (see Section 2) and $E$ is a finite dimensional representation of the Lie algebra of $H$ such that the tensor product $S \otimes E$ lifts to a representation of $H$. This induces a homogeneous vector bundle, denoted by $\mathcal{S} \otimes \mathcal{E}$, over $G / H$. Acting on the space $C^{\infty}(G / H, \mathcal{S} \otimes \mathcal{E})$ of smooth sections, there is the cubic Dirac operator

$$
\mathcal{D}(E): C^{\infty}(G / H, \mathcal{S} \otimes \mathcal{E}) \rightarrow C^{\infty}(G / H, \mathcal{S} \otimes \mathcal{E}) .
$$

$\mathcal{D}(E)$ is a $G$-invariant differential operator equal to the sum of the above first-order term $\widehat{\mathcal{D}}(E)$ and a zero-order term, which comes from a degree three element $\mathbf{c}$ in the Clifford algebra of 
the orthogonal complement of the complexification of the Lie algebra of $H$. The cubic term c is defined as the image, via the Chevalley isomorphism, of the 3 -form

$$
(X, Y, Z) \in\left(T_{e H} G / H\right)^{3} \mapsto\langle X,[Y, Z]\rangle .
$$

The reason we have to consider cubic Dirac operators for general spacetimes $G / H$ is because the square of the ordinary Dirac operator $\widehat{\mathcal{D}}(E)$ need not differ from the Laplacian by a constant as it should be. Note that when $H$ is a symmetric subgroup, the zero-order term vanishes and the cubic Dirac operator $\mathcal{D}(E)$ coincides with the ordinary Dirac operator $\widehat{\mathcal{D}}(E)$.

The kernel of (twisted) Dirac operators plays an important role in representation theory. When $G$ admits discrete series representations and $H$ is a maximal compact subgroup in $G$, then the $L^{2}$-kernel of $\mathcal{D}(E)$ is an irreducible unitary representation belonging to the discrete series of $G$ and every discrete series representation of $G$ is the $L^{2}$-kernel of $\mathcal{D}(E)$ for some $E$ [AS77, Par72]. When $H$ is not compact, the smooth kernel of $\mathcal{D}(E)$ contains a principal series representation (now $G$ and $H$ need not be equal rank). This is done in [MZ14b] by constructing an intertwining map $\mathcal{P}_{\mathcal{E}}$ analogous to the Poisson transform:

$$
\mathcal{P}_{\mathcal{E}}: C^{\infty}(G / P, \mathcal{W}) \rightarrow C^{\infty}(G / H, \mathcal{S} \otimes \mathcal{E})
$$

where $P$ is a parabolic subgroup of $G$ and $W$ a representation of $P$. It turns out that discrete series representations and principal series representations are building blocks of the representation theory of real reductive Lie groups, since most representations can be embedded in a principal series representation by Casselman embedding Theorem.

Our main result (Theorem 4.1) provides a systematic process that produces Weyl fermions on $G / H$ from Weyl fermions lying in the image of the Poisson transform $\mathcal{P}$, via a commutative diagram of the following form

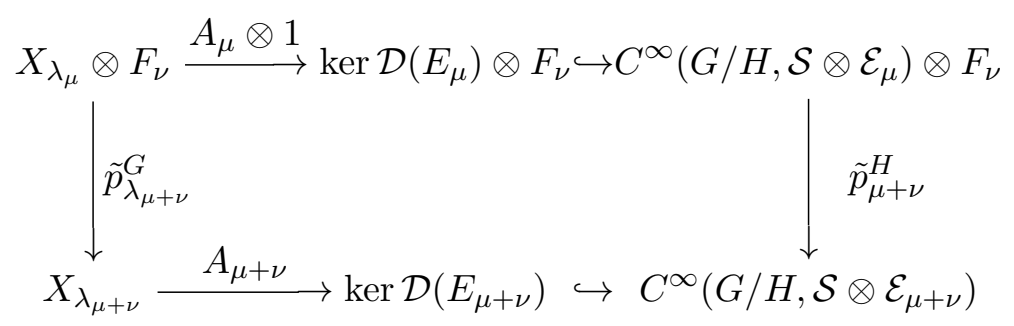

where ker $\mathcal{D}\left(E_{\bullet}\right)$ denotes the smooth kernel of $\mathcal{D}\left(E_{\bullet}\right),\left\{X_{\lambda_{\mu}}\right\}$ is a family of smooth representations of $G$ with infinitesimal character $\lambda_{\mu}$ and $F_{\nu}$ (resp. $E_{\mu}$ ) is a finite dimensional irreducible representation of $G$ (resp. $H$ ) with highest weight $\nu$ (resp. $\mu$ ) with respect to some fixed positive systems of roots. The various maps $A$ • are $G$-intertwining operators, 
and the maps $\tilde{p}_{\bullet}^{G}$ and $\tilde{p}_{\bullet}^{H}$ are the projections on the corresponding infinitesimal characters with respect to $G$ and $H$. We are mainly concerned with the case where the representations $X_{\lambda_{\mu}}$ are principal series representations and the intertwining maps $A_{\bullet}$ are Poisson-type maps $\mathcal{P}$. However, due to many stages required in the construction of the Poisson maps $\mathcal{P}$, other families of representations have to be considered first, including the case of compact groups and fundamental series representations. In each case, we obtain an intertwining map between the family of representations and the kernel of the Dirac operator, as well as a diagram of the form (6).

In other words, given a Weyl fermion interacting with a particle living in the bundle $\mathcal{E}_{\mu}$, the interaction of this fermion with a particle living in the bundle $\mathcal{F}_{\nu}$ contains a Weyl fermion interacting with a particle in the bundle $\mathcal{E}_{\mu+\nu}$. Moreover, the action of the Casimir operator of $G$ (resp. $H$ ) on $F_{\bullet}$ (resp. $E_{\bullet}$ ) induces some energy level restriction on the Weyl fermions.

The paper is organized as follows. The definition and the main properties of (cubic) Dirac operators are recalled in Section 1. Several aspects of the kernel of Dirac operators are discussed in Section 2 such as the translation principle for compact groups. Section 3 is devoted to the construction of various commutative diagrams which are used to prove that a specific embedding of fundamental series representations into the Dirac kernel commutes with translation functors. Finally, in Section 4, we show that the intertwining map defined in [MZ14b], which produces explicit non-zero solutions to Weyl equations $\mathcal{D}\left(E_{\mu}\right)=0$ on $G / H$, commutes with translations by finite dimensional representations (Theorem 4.1). This provides a systematic geometric process which produces interacting Weyl fermions with a fixed energy level on the homogeneous spacetime $G / H$ as follows:

- (non zero) Weyl fermions $f$ on the spacetime $G / H$, interacting with particles 'living' in the bundle $\mathcal{E}_{\mu}$ (i.e. $\mathcal{D}\left(E_{\mu}\right) f=0$ ), can be described as the 'Poisson' transform $\mathcal{P}_{\mathcal{E}_{\mu}}$ of some (non zero) section over the piece $G / P$ of the 'boundary' of $G / H$,

- the Weyl fermion $f$ can interact with particles in the bundle $\mathcal{F}_{\nu}$ and the map $\tilde{p}_{\mu+\nu}^{H}$ above produces a composite object consisting of a combination of (non zero) Weyl fermions over $G / H$,

- this composite object itself can be described as the image by the 'Poisson' transform $\mathcal{P}_{\mathcal{E}_{\mu+\nu}}$ of some (non zero) section over the 'boundary' of $G / H$.

We thank the anonymous referee for his/her thorough review and highly appreciate the comments and suggestions, which significantly contributed to improving the text. 


\section{Dirac operators}

Let $G$ be a connected reductive Lie group with complexified Lie algebra $\mathfrak{g}$ and Killing form $B$. Let $H$ be a connected reductive subgroup of $G$ with complexified Lie algebra $\mathfrak{h}$. Let $\mathcal{U}(\mathfrak{g})$ (resp. $\mathcal{U}(\mathfrak{h}))$ be the universal enveloping algebra of $\mathfrak{g}$ (resp. $\mathfrak{h}$ ). We assume that the restriction of $B$ to $\mathfrak{h} \times \mathfrak{h}$ is still non degenerate. We have an orthogonal $H$-invariant decomposition of $\mathfrak{g}$

$$
\mathfrak{g}=\mathfrak{h} \oplus \mathfrak{h}^{\perp} .
$$

In particular, the restriction of $B$ to $\mathfrak{h}^{\perp}$ remains non degenerate. The homogeneous reductive space $G / H$ has complexified tangent space at the origin canonically identified with $\mathfrak{h}^{\perp}$. We consider the Clifford algebra $\mathrm{Cl}\left(\mathfrak{h}^{\perp}\right)$ of $\mathfrak{h}^{\perp}$ with respect to $\left.B\right|_{\mathfrak{h}^{\perp} \times \mathfrak{h}^{\perp}}$. The defining relations of the Clifford algebra are:

$$
X X^{\prime}+X^{\prime} X=-2 B\left(X, X^{\prime}\right) \quad\left(\forall X, X^{\prime} \in \mathfrak{h}^{\perp}\right) .
$$

The restriction of the adjoint action of $G$ to $H$ leaves the space $\mathfrak{h}^{\perp}$ stable and preserves $B$. In other words, we have a group homomorphism

$$
\left.\operatorname{Ad}\right|_{H}: H \rightarrow \mathrm{SO}\left(\mathfrak{h}^{\perp},\left.B\right|_{\mathfrak{h}^{\perp} \times \mathfrak{h}^{\perp}}\right) .
$$

Write $S_{\mathfrak{g} / \mathfrak{h}}$ for the exterior algebra of a maximal isotropic subspace in $\mathfrak{h}^{\perp}$. A specific choice of an isotropic subspace is made in [MZ14b, Section 1.3.1]. Consider the Clifford multiplication

$$
\gamma: \operatorname{Cl}\left(\mathfrak{h}^{\perp}\right) \longrightarrow \operatorname{End}\left(S_{\mathfrak{g} / \mathfrak{h}}\right) .
$$

Then the spin representation $\left(S_{\mathfrak{g} / \mathfrak{h}}, s_{\mathfrak{g} / \mathfrak{h}}\right)$ of $\mathfrak{h}$ is defined as the composition map

$$
\mathfrak{h} \stackrel{\left.\operatorname{ad}\right|_{\mathfrak{h}}}{\hookrightarrow} \mathfrak{s o}\left(\mathfrak{h}^{\perp},\left.B\right|_{\mathfrak{h}} ^{\perp} \times \mathfrak{h}^{\perp}\right) \hookrightarrow \operatorname{Cl}\left(\mathfrak{h}^{\perp}\right) \stackrel{\gamma}{\longrightarrow} \operatorname{End}\left(S_{\mathfrak{g} / \mathfrak{h}}\right) .
$$

Let $(E, \sigma)$ be a finite-dimensional representation of $\mathfrak{h}$ such that the tensor product representation $S_{\mathfrak{g} / \mathfrak{h}} \otimes E$ lifts to a representation of the group $H$. We have a finite rank complex vector bundle

$$
\mathcal{S}_{\mathfrak{g} / \mathfrak{h}} \otimes \mathcal{E}=G \times_{H}\left(S_{\mathfrak{g} / \mathfrak{h}} \otimes E\right)
$$

over $G / H$, whose space of smooth sections $C^{\infty}\left(G / H, \mathcal{S}_{\mathfrak{g} / \mathfrak{h}} \otimes \mathcal{E}\right)$ will be identified with the space $C^{\infty}\left(G, S_{\mathfrak{g} / \mathfrak{h}} \otimes E\right)^{H}$ of smooth $S_{\mathfrak{g} / \mathfrak{h}} \otimes E$-valued maps $f$ on $G$ satisfying the equivariance condition:

$$
f(g h)=h^{-1} \cdot f(g) \quad(\forall h \in H, g \in G) .
$$

From now on, if $F$ is a finite dimensional representation of $H$ we will denote by $\mathcal{F}$ the induced vector bundle of $G / H$. 
Let $\sharp: \mathcal{U}(\mathfrak{h}) \rightarrow \mathcal{U}(\mathfrak{h})$ be the antipode. This is the antiautomorphism of $\mathcal{U}(\mathfrak{h})$ given by $Y^{\sharp}=-Y$ on $\mathfrak{h}$, so that

$$
\left(Y_{1} \cdots Y_{n}\right)^{\sharp}=(-1)^{n} Y_{n} \cdots Y_{1} \quad\left(\forall Y_{1}, \cdots, Y_{n} \in \mathfrak{h}\right) .
$$

We consider $\mathcal{U}(\mathfrak{g})$ as a right $\mathcal{U}(\mathfrak{h})$-module and $\operatorname{End}\left(S_{\mathfrak{g} / \mathfrak{h}} \otimes E\right)$ as the left $\mathcal{U}(\mathfrak{h})$-module given by

$$
v \cdot T=T \circ\left(s_{\mathfrak{g} / \mathfrak{h}} \otimes \sigma\right)\left(v^{\sharp}\right) \quad\left(\forall v \in \mathcal{U}(\mathfrak{h}), T \in \operatorname{End}\left(S_{\mathfrak{g} / \mathfrak{h}} \otimes E\right)\right) .
$$

Let $J$ be the left ideal of $\left(\mathcal{U}(\mathfrak{g}) \otimes \operatorname{End}\left(S_{\mathfrak{g} / \mathfrak{h}} \otimes E\right)\right)$ generated by elements of the form

$$
u v \otimes T-u \otimes v \cdot T \quad\left(\forall u \in \mathcal{U}(\mathfrak{g}), v \in \mathcal{U}(\mathfrak{h}), T \in \operatorname{End}\left(S_{\mathfrak{g} / \mathfrak{h}} \otimes E\right)\right) .
$$

Then the tensor product over $\mathcal{U}(\mathfrak{h})$ is defined as the quotient

$$
\mathcal{U}(\mathfrak{g}) \underset{\mathcal{U}(\mathfrak{h})}{\otimes} \operatorname{End}\left(S_{\mathfrak{g} / \mathfrak{h}} \otimes E\right)=\left(\mathcal{U}(\mathfrak{g}) \otimes \operatorname{End}\left(S_{\mathfrak{g} / \mathfrak{h}} \otimes E\right)\right) / J
$$

Let $q$ be the quotient map. The action of $H$ on the tensor product leaves $J$ stable, and hence induces an action of $H$ on the quotient. The invariant space for this action is the image of the $H$-invariants in the tensor product under $q$ so that

$$
q:\left(\mathcal{U}(\mathfrak{g}) \otimes \operatorname{End}\left(S_{\mathfrak{g} / \mathfrak{h}} \otimes E\right)\right)^{H} \rightarrow\left(\mathcal{U}(\mathfrak{g}) \underset{\mathcal{U}(\mathfrak{h})}{\otimes} \operatorname{End}\left(S_{\mathfrak{g} / \mathfrak{h}} \otimes E\right)\right)^{H}
$$

It is easy to check that the space $\left(\mathcal{U}(\mathfrak{g}) \otimes_{\mathcal{U}(\mathfrak{h})} \operatorname{End}\left(S_{\mathfrak{g} / \mathfrak{h}} \otimes E\right)\right)^{H}$ is an algebra. For $X \in \operatorname{Lie}(G)$, let $r(X)$ denote the left $G$-invariant differential operator on $G$ given by right differentiation:

$$
(r(X) f)(g)=\left[\frac{d}{d t} f(g \exp (t X))\right]_{t=0} \quad \forall f \in C^{\infty}(G) .
$$

Extend complex-linearly $r$ to the complexification $\mathfrak{g}$ of $\operatorname{Lie}(G)$.

Proposition 1.1. [KR00] The algebra $\mathbb{D}_{G}\left(\mathcal{S}_{\mathfrak{g} / \mathfrak{h}} \otimes \mathcal{E}\right)$ of $G$-invariant differential operators acting on (smooth) sections of $\mathcal{S}_{\mathfrak{g} / \mathfrak{h}} \otimes \mathcal{E}$ is isomorphic to the algebra $\left(\mathcal{U}(\mathfrak{g}) \otimes \mathcal{U}(\mathfrak{h}) \operatorname{End}\left(S_{\mathfrak{g} / \mathfrak{h}} \otimes E\right)\right)^{H}$. This isomorphism is induced by the following representation of $\mathcal{U}(\mathfrak{g}) \otimes \operatorname{End}\left(S_{\mathfrak{g} / \mathfrak{h}} \otimes E\right)$ on $C^{\infty}\left(G, S_{\mathfrak{g} / \mathfrak{h}} \otimes E\right):$

$$
(X \otimes T) f(g)=T(r(X) f(g)) \quad\left(\forall X \in \mathfrak{g}, T \in \operatorname{End}\left(S_{\mathfrak{g} / \mathfrak{h}} \otimes E\right), f \in C^{\infty}\left(G, S_{\mathfrak{g} / \mathfrak{h}} \otimes E\right)\right) .
$$

Let $\mathbf{c}$ be the degree three element in $\mathrm{Cl}\left(\mathfrak{h}^{\perp}\right)$ defined as the image under the Chevalley isomorphism of the 3 -form on $\mathfrak{h}$ given by

$$
(X, Y, Z) \mapsto B(X,[Y, Z]) .
$$

Letting $\left\{X_{j}\right\}$ be an orthonormal basis of $\mathfrak{h}^{\perp}$ with respect to $B$, the geometric cubic Dirac operator is the element $\mathcal{D}_{G / H}(E)$ (or $\mathcal{D}(E)$ when no confusion is possible) of $\mathbb{D}_{G}\left(\mathcal{S}_{\mathfrak{g} / \mathfrak{h}} \otimes \mathcal{E}\right.$ ) 
defined by

$$
\mathcal{D}_{G / H}(E)=\sum_{j} r\left(X_{j}\right) \otimes \gamma\left(X_{j}\right) \otimes 1+1 \otimes \gamma(\mathbf{c}) \otimes 1
$$

with

$$
\mathbf{c}=\sum_{j<k<\ell} B\left(X_{j},\left[X_{k}, X_{\ell}\right]\right) X_{j} X_{k} X_{\ell}
$$

When $H$ is a symmetric subgroup of $G$, then $\left[\mathfrak{h}^{\perp}, \mathfrak{h}^{\perp}\right] \subset \mathfrak{h}$ and $\mathbf{c}=0$, i.e., the cubic Dirac operator $\mathcal{D}_{G / H}(E)$ coincides with the usual Dirac operator $\widehat{\mathcal{D}}_{G / H}(E)$ :

$$
\widehat{\mathcal{D}}_{G / H}(E)=\sum_{j} r\left(X_{j}\right) \otimes \gamma\left(X_{j}\right) \otimes 1 .
$$

Both $\mathcal{D}_{G / H}(E)$ and $\widehat{\mathcal{D}}_{G / H}(E)$ define $G$-invariant differential operators and their above expressions are independent of the choice of the basis $\left\{X_{j}\right\}$.

Since the space $S_{\mathfrak{g} / \mathfrak{h}} \otimes E$ is also a Clifford module, we may consider the representation

$$
I \otimes\left(\gamma \otimes I_{E}\right): \mathcal{U}(\mathfrak{g}) \otimes \operatorname{Cl}\left(\mathfrak{h}^{\perp}\right) \rightarrow \mathcal{U}(\mathfrak{g}) \otimes \operatorname{End}\left(S_{\mathfrak{g} / \mathfrak{h}} \otimes E\right) .
$$

It sends $H$-invariants to $H$-invariants and goes down to an algebra homomorphism

$$
\varphi_{E}:\left(\mathcal{U}(\mathfrak{g}) \otimes \operatorname{Cl}\left(\mathfrak{h}^{\perp}\right)\right)^{H} \stackrel{q \circ\left(I \otimes \gamma \otimes I_{E}\right)}{\longrightarrow}\left(\mathcal{U}(\mathfrak{g}) \underset{\mathcal{U}(\mathfrak{h})}{\otimes} \operatorname{End}\left(S_{\mathfrak{g} / \mathfrak{h}} \otimes E\right)\right)^{H} .
$$

As already mentioned in the introduction, one of the most important facts about Dirac operator is that its square has a very simple expression. Let

$$
\widehat{D}_{\mathfrak{g} / \mathfrak{h}}=\sum_{j} X_{j} \otimes X_{j} \in\left(\mathcal{U}(\mathfrak{g}) \otimes \operatorname{Cl}\left(\mathfrak{h}^{\perp}\right)\right)^{H} \text { and } D_{\mathfrak{g} / \mathfrak{h}}=\widehat{D}_{\mathfrak{g} / \mathfrak{h}}+1 \otimes \mathbf{c}
$$

such that $\varphi_{E}\left(D_{\mathfrak{g} / \mathfrak{h}}\right)=\mathcal{D}_{G / H}(E)$. Let $\delta$ be the Lie algebra homomorphism defined by

$$
\begin{aligned}
\delta: \mathcal{U}(\mathfrak{h}) & \longrightarrow \mathcal{U}(\mathfrak{g}) \otimes \mathrm{Cl}\left(\mathfrak{h}^{\perp}\right) \\
X & \longmapsto X \otimes 1+1 \otimes X .
\end{aligned}
$$

Let $\Omega_{G}$ be the Casimir operator for $G$ is defined as the image of the identity element through the sequence of $G$-equivariant maps

$$
\operatorname{Hom}_{\mathbb{C}}(\mathfrak{g}, \mathfrak{g}) \longrightarrow \mathfrak{g} \otimes \mathfrak{g}^{\star} \longrightarrow \mathfrak{g} \otimes \mathfrak{g} \hookrightarrow T(\mathfrak{g}) \longrightarrow \mathcal{U}(\mathfrak{g}),
$$

where $\mathfrak{g}^{\star}$ denotes the vector dual of $\mathfrak{g}$ and $T(\mathfrak{g})$ the tensor algebra of $\mathfrak{g}$. The Casimir operator $\Omega_{H}$ of $H$ is defined in a similar way.

Theorem 1.2. [Kos99, Par72] There exist constants $c_{\mathfrak{g}}$ and $c_{\mathfrak{h}}$ depending on $\mathfrak{g}$ and $\mathfrak{h}$ respectively such that

$$
D_{\mathfrak{g} / \mathfrak{h}}^{2}=\left(\Omega_{G}+c_{\mathfrak{g}}\right)-\delta\left(\Omega_{H}+c_{\mathfrak{h}}\right)
$$


A short proof was given in [Pru10], it avoids the computation of the constants $c_{\mathfrak{g}}$ and $c_{\mathfrak{h}}$ which are explicitly calculated in [Kos99, Par72].

There is an algebraic analog of the geometric Dirac operator $\mathcal{D}_{G / H}(E)$. Let $(X, \pi)$ be a smooth representation of $G$. The algebraic Dirac operator is the element

$$
D_{X}(E)=\left(\pi \otimes \gamma \otimes I_{E}\right)\left(D_{\mathfrak{g} / \mathfrak{h}}\right) \in \operatorname{End}\left(X \otimes S_{\mathfrak{g} / \mathfrak{h}} \otimes E\right)
$$

defined as the image of $D_{\mathfrak{g} / \mathfrak{h}}$ by the map $\pi \otimes \gamma \otimes I_{E}$. In the same way one obtains the operator $\widehat{D}_{X}(E)$. Note that in the case when $E=\mathbb{C}, H=\{e\}$ and $\pi=1_{G}$ is the trivial representation of $G$, one gets that $c_{\mathfrak{g}}=s_{\mathfrak{g}}\left(\Omega_{G}\right)$. So, since the infinitesimal character of $S_{\mathfrak{g}}$ is half the sum of positive roots $\rho_{\mathfrak{g}}$ (assume a system of positive roots has been fixed), we have $c_{\mathfrak{g}}=\left\|\rho_{\mathfrak{g}}\right\|^{2}$. We also note $D_{X}:=D_{X}\left(1_{H}\right)$ the usual algebraic cubic Dirac operator on $X$.

The Kostant-Parthasarathy formula for the square of $D_{X}(E)$ reads as (see [Kos99])

$$
D_{X}(E)^{2}=\pi\left(\Omega_{G}\right) \otimes 1 \otimes 1-\pi \otimes s_{\mathfrak{g} / \mathfrak{h}}\left(\Omega_{H}\right) \otimes 1+\left\|\rho_{\mathfrak{g}}\right\|^{2}-\left\|\rho_{\mathfrak{h}}\right\|^{2} .
$$

In the sequel we will use the notation $\rho$ • above in several situations. Let $\mathfrak{q}^{\prime}$ be a complex Lie subalgebra of a complex Lie algebra $\mathfrak{q}$. Suppose $\mathfrak{t}^{\prime}$ is a Cartan subalgebra of $\mathfrak{q}^{\prime}$ contained in a Cartan subalgebra $\mathfrak{t}$ of $\mathfrak{q}$ such that $\mathfrak{t}=\mathfrak{t}^{\prime}+\mathfrak{t}^{\prime \prime}$, for some fixed vector subspace $\mathfrak{t}^{\prime \prime}$. Choose positive systems $\Delta^{+}(\mathfrak{t}, \mathfrak{q})$ and $\Delta^{+}\left(\mathfrak{t}^{\prime}, \mathfrak{q}^{\prime}\right)$ of roots in $\mathfrak{q}$ and $\mathfrak{q}^{\prime}$ respectively, with the compatibility condition that

$$
\Delta^{+}\left(\mathfrak{t}^{\prime}, \mathfrak{q}^{\prime}\right)=\left\{\left.\alpha\right|_{\mathfrak{t}^{\prime}} \in \Delta\left(\mathfrak{t}^{\prime}, \mathfrak{q}^{\prime}\right), \alpha \in \Delta^{+}(\mathfrak{t}, \mathfrak{q}), \mathfrak{q}^{(\alpha)} \subset \mathfrak{q}^{\prime},\left.\alpha\right|_{\mathfrak{t}^{\prime}} \neq 0\right\}
$$

where $\mathfrak{q}^{(\alpha)}$ is the $\alpha$-root space. Then $\rho_{\mathfrak{q}}\left(\right.$ resp. $\left.\rho_{\mathfrak{q}^{\prime}}\right)$ is half the sum of the elements of $\Delta^{+}(\mathfrak{t}, \mathfrak{q})$ (resp. $\Delta^{+}\left(\mathfrak{t}^{\prime}, \mathfrak{q}^{\prime}\right)$ ), while $\rho_{\mathfrak{q}^{\prime}}$ is defined as $\rho_{\mathfrak{q}}-\rho_{\mathfrak{q}^{\prime}}$.

\section{Kernel of Dirac operators and translations}

2.1. Tensoring with finite dimensional representations. We keep the previous notations and we let $(F, \pi)$ (resp. $E$ ) be a finite dimensional representation of $G$ (resp. $H$ ). One easily checks the following two properties.

Proposition 2.1. The map $\alpha^{H}: C^{\infty}\left(G / H, \mathcal{S}_{\mathfrak{g} / \mathfrak{h}} \otimes \mathcal{E}\right) \otimes F \rightarrow C^{\infty}\left(G / H, \mathcal{S}_{\mathfrak{g} / \mathfrak{h}} \otimes \mathcal{E} \otimes \mathcal{F}\right)$ given by

$$
\alpha^{H}(f \otimes w)(g)=f(g) \otimes \pi\left(g^{-1}\right) \cdot w \in S_{\mathfrak{g} / \mathfrak{h}} \otimes E \otimes F
$$

is a smooth G-module isomorphism.

The second property relates the various Dirac operators on the bundles in consideration. 
Proposition 2.2. One has

$$
\alpha^{H} \circ\left(\mathcal{D}_{G / H}(E) \otimes I_{F}\right)=\left(\mathcal{D}_{G / H}(E \otimes F)+1 \otimes \widehat{D}_{F}(E)\right) \circ \alpha^{H} .
$$

2.2. The compact group case. We will need to describe $\operatorname{ker} \mathcal{D}_{K / H}(E)$ when $K$ is compact (with complexified Lie algebra $\mathfrak{k}$ ) and $E$ is an irreducible representation of $H$. This has been done explicitly by Landweber [Lan00] when $K$ and $H$ have the same (complex) rank. The general case has been studied in the algebraic framework by Mehdi and Zierau in [MZ06]. If $V$ is an irreducible representation of $K$, and $E^{\prime}$ is a highest weight representation of $H$ in $E \otimes V$, we will need a precise relation between the $K$-modules $\operatorname{ker} \mathcal{D}_{K / H}\left(E^{\prime}\right)$ and $\operatorname{ker} \mathcal{D}_{K / H}(E) \otimes V$.

Let $\mathfrak{t}$ be a Cartan subalgebra of $\mathfrak{k}$ containing a Cartan subalgebra $\mathfrak{t}_{\mathfrak{h}}$ of $\mathfrak{h}$. There is a subspace $\mathfrak{t}_{\mathfrak{h} \perp}$ of $\mathfrak{h}^{\perp}$ such that $\mathfrak{t}=\mathfrak{t}_{\mathfrak{h}}+\mathfrak{t}_{\mathfrak{h} \perp}$. Let $\Delta^{+} \subset \Delta(\mathfrak{t}, \mathfrak{k})$ be a positive root system defined by a lexicographic order with $\mathfrak{t}_{\mathfrak{h}}$ first, then $\mathfrak{t}_{\mathfrak{h} \perp}$. Such a positive system has the property that

$$
\Delta^{+}(\mathfrak{h}):=\left\{\left.\alpha\right|_{\mathfrak{t}_{\mathfrak{h}}}, \mathfrak{k}^{(\alpha)} \subset \mathfrak{h}, \alpha \in \Delta^{+} \text {and }\left.\alpha\right|_{\mathfrak{t}_{\mathfrak{h}}} \neq 0\right\}
$$

is a positive system of roots in $\mathfrak{h}$. Here $\mathfrak{k}^{(\alpha)}$ is the $\alpha$-root space in $\mathfrak{k}$. Let $\rho_{\mathfrak{k}}$ (resp. $\rho_{\mathfrak{h}}$ ) be half the sum of positive roots in $\Delta^{+}$(resp. $\left.\Delta^{+}(\mathfrak{h})\right)$ and $W(K)$ be the Weyl group of $K$. Let $d$ be the largest integer less than or equal to $\frac{1}{2}(\operatorname{rank} \mathfrak{k}-\operatorname{rank} \mathfrak{h})$.

Theorem 2.3. Let $E_{\mu}$ be an irreducible representation of $H$ with highest weight $\mu$ with respect to $\Delta^{+}(\mathfrak{h})$. The following assertions are equivalent:

(i) there exists $w \in W(K)$ such that $\xi_{\mu}=w\left(\mu+\rho_{\mathfrak{h}}\right)-\rho_{\mathfrak{k}} \in \mathfrak{t}^{*}$ is $\Delta^{+}$-dominant analytically integral;

(ii) there exists a unique $w \in W(K)$ such that $\xi_{\mu}=w\left(\mu+\rho_{\mathfrak{h}}\right)-\rho_{\mathfrak{k}} \in \mathfrak{t}^{*}$ is $\Delta^{+}$-dominant analytically integral;

(iii) $\operatorname{ker} \mathcal{D}_{K / H}\left(E_{\mu}\right) \neq\{0\}$.

If one of (therefore, any of) the equivalent conditions holds, then

$$
\operatorname{ker} \mathcal{D}_{K / H}\left(E_{\mu}\right) \simeq 2^{d} V_{\xi_{\mu}}
$$

Proof. As $K$ is compact we may assume that any finite dimensional representation $V$ of $K$ is unitary. Then there is a canonical isomorphism between $V$ and its dual $V^{*}$. Using this isomorphism and [MZ14b, Equation (1.10)] we get, for any irreducible representation $V_{\nu}$ of $K$ with highest weight $\nu$, an isomorphism

$$
\operatorname{Hom}_{K}\left(V_{\nu}, \operatorname{ker} \mathcal{D}_{K / H}\left(E_{\mu}\right)\right) \simeq \operatorname{Hom}_{H}\left(\operatorname{ker} D_{V_{\nu}}, E_{\mu}\right) .
$$

Now, by [MZ14a], ker $D_{V_{\nu}}$ can be described as follows. Letting

$$
W_{1}(\nu):=\left\{w \in W(K),\left.w\left(\nu+\rho_{\mathfrak{k}}\right)\right|_{\mathfrak{t}_{\mathfrak{h} \perp}}=0 \text { and }\left.w\left(\nu+\rho_{\mathfrak{k}}\right)\right|_{\mathfrak{t}_{\mathfrak{h}}} \text { is } \Delta^{+}(\mathfrak{h})-\text { dominant }\right\}
$$


we obtain that

$$
\operatorname{ker} D_{V_{\nu}}=\oplus_{w \in W_{1}(\nu)} 2^{d} E_{w\left(\nu+\rho_{\mathfrak{k}}\right)-\rho_{\mathfrak{h}}} .
$$

It follows that

$$
\operatorname{ker} \mathcal{D}_{K / H}\left(E_{\lambda}\right)=\oplus_{\nu} 2^{d} V_{\nu}
$$

where the sum is over all $\nu$ such that there exists $w \in W_{1}(\nu)$ such that $\nu+\rho_{\mathfrak{k}}=w^{-1}\left(\lambda+\rho_{\mathfrak{h}}\right)$. Note that if such $\nu$ exists it is necessarily unique. In fact we would have $w\left(\nu^{\prime}+\rho_{\mathfrak{k}}\right)=\nu+\rho_{\mathfrak{k}}$. But $\nu+\rho_{\mathfrak{g}}$ is in the open Weyl chamber, so this implies that $w=1$ and $\nu^{\prime}=\nu$.

Now we need to make a choice of

$$
b_{\mu} \in \operatorname{Hom}_{K}\left(V_{\xi_{\mu}}, \operatorname{ker} \mathcal{D}_{K / H}\left(E_{\mu}\right)\right) .
$$

Let $\mu$ be an analytically integral weight in $\mathfrak{t}_{\mathfrak{h}}$ which is dominant for some positive system of roots in $\mathfrak{h}$. We specify $\Delta^{+}$by choosing $\mu$ as the first basis vector and define $\Delta^{+}(\mathfrak{h})$ as above. Then $\mu$ is dominant for both $\Delta^{+}$and $\Delta^{+}(\mathfrak{h})$. Let $E_{\mu}$ be an irreducible finite dimensional representation of $H$ with highest weight $\mu$. Let $\rho_{\mathfrak{h} \perp}=\rho_{\mathfrak{g}}-\rho_{\mathfrak{h}}$ and let $\xi_{\mu} \in \mathfrak{t}^{*}$ be defined as

$$
\xi_{\mu}=\mu-\rho_{\mathfrak{h} \perp} .
$$

In particular, $\left.\xi_{\mu}\right|_{\mathfrak{t}_{\mathfrak{h}}}=\mu+\rho_{\mathfrak{h}}-\left.\rho_{\mathfrak{g}}\right|_{\mathfrak{t}_{\mathfrak{h}}}$ and $\left.\xi_{\mu}\right|_{\mathfrak{t}_{\mathfrak{h} \perp}}=-\left.\rho_{\mathfrak{g}}\right|_{\mathfrak{t}_{\mathfrak{h} \perp}}$. Let $V_{\xi_{\mu}}$ be the irreducible representation of $K$ with highest weight $\xi_{\mu}$. By the previous proposition there exists a non zero $b_{\mu}: V_{\xi_{\mu}} \rightarrow \operatorname{ker} \mathcal{D}_{K / H}\left(E_{\mu}\right)$. We choose a specific $b_{\mu}$ as follows. First, observe that there exists a non zero $H$-invariant vector $v$ in $S_{\mathfrak{k} / \mathfrak{h}} \otimes E_{\rho_{\mathfrak{h} \perp}}$ and a non zero $H$-equivariant map

$$
\begin{array}{ccccc}
E_{\xi_{\mu} \mid \mathfrak{t}_{\mathfrak{h}}} & \rightarrow & \left(S_{\mathfrak{k} / \mathfrak{h}} \otimes E_{\rho_{\mathfrak{h} \perp}}\right) & \otimes & E_{\left.\xi_{\mu}\right|_{\mathfrak{t}_{\mathfrak{h}}}} \\
w & \mapsto & v & \otimes & w .
\end{array}
$$

Then composing with the Cartan projection $E_{\rho_{\mathfrak{h} \perp}} \otimes E_{\left.\xi_{\mu}\right|_{\mathfrak{h}}} \rightarrow E_{\mu}$, this yields a homomorphism

$$
E_{\left.\xi_{\mu}\right|_{\mathfrak{h}}} \rightarrow S_{\mathfrak{k} / \mathfrak{h}} \otimes E_{\mu} .
$$

The projection of the restriction $\left.V_{\xi_{\mu}}\right|_{H}$ of $V_{\xi_{\mu}}$ to $H$ onto its highest weight component $E_{\left.\xi_{\mu}\right|_{\mathfrak{h}}}$ defines a non-zero element of $\operatorname{Hom}_{H}\left(\left.V_{\xi_{\mu}}\right|_{H}, E_{\left.\xi_{\mu}\right|_{\mathfrak{t}_{\mathfrak{h}}}}\right)$. By Frobenius reciprocity, we get a nonzero element in $\operatorname{Hom}_{K}\left(V_{\xi_{\mu}}, C^{\infty}\left(K / H, \mathcal{E}_{\xi_{\mu}||_{\mathfrak{h}}}\right)\right)$. We obtain a $K$-homomorphism by composition

$$
b_{\mu}: V_{\xi_{\mu}} \rightarrow C^{\infty}\left(K / H, \mathcal{E}_{\left.\xi_{\mu}\right|_{\mathfrak{h}}}\right) \rightarrow C^{\infty}\left(K / H, \mathcal{S}_{\mathfrak{k} / \mathfrak{h}} \otimes \mathcal{E}_{\mu}\right) .
$$

Lemma 2.4. The range of $b_{\mu}$ is contained in the kernel of the Dirac operator $\mathcal{D}_{K / H}\left(E_{\mu}\right)$.

Proof. For any finite dimensional unitary representations $V$ of $K$, and $E$ of $H$, we have an isomorphism (see (1.10) in [MZ14b])

$$
\phi: \operatorname{Hom}_{K}\left(V, C^{\infty}\left(K / H, \mathcal{S}_{\mathfrak{k} / \mathfrak{h}} \otimes \mathcal{E}\right)\right) \stackrel{\sim}{\longrightarrow} \operatorname{Hom}_{H}\left(E, V \otimes S_{\mathfrak{k} / \mathfrak{h}}\right)
$$


such that, for $T \in \operatorname{Hom}_{K}\left(V, C^{\infty}\left(K / H, \mathcal{S}_{\mathfrak{k} / \mathfrak{h}} \otimes \mathcal{E}\right)\right)$,

$$
T(V) \subset \operatorname{ker} \mathcal{D}_{K / H}(E) \text { if and only if } \phi(T)(E) \subset \operatorname{ker} D_{V}
$$

The proof now follows from (9).

Let $E$ be a finite dimensional representation of $H$. Assume that $E$ contains an irreducible $H$-representation $E_{\nu}$ with highest weight $\nu$ (with respect to $\Delta^{+}(\mathfrak{h})$ ) which occurs with multiplicity one. We denote by $p_{\nu}^{H}$ the $H$-equivariant projection on the highest weight component

$$
E \stackrel{p_{\nu}^{H}}{\longrightarrow} E_{\nu}
$$

In a similar way, we define the $K$-equivariant projection $p_{\bullet}^{K}$. Note that when $E$ is the tensor product $E_{\mu} \otimes E_{\nu}$, then $E$ contains an irreducible component $E_{\lambda+\nu}$ with highest weight $\mu+\nu$ which coincides with the Cartan component. In this case, the map $p_{\nu}^{H}$ coincides with the Cartan projection and it induces a smooth $K$-equivariant map (denoted by the same symbol)

$$
C^{\infty}\left(K / H, \mathcal{S}_{\mathfrak{k} / \mathfrak{h}} \otimes \mathcal{E}_{\mu} \otimes \mathcal{V}_{\nu}\right) \stackrel{p_{\mu+\nu}^{H}}{\longrightarrow} C^{\infty}\left(K / H, \mathcal{S}_{\mathfrak{k} / \mathfrak{h}} \otimes \mathcal{E}_{\mu+\nu}\right)
$$

We shall always assume that $E_{\mu}$ is chosen in such a way that the $\mathfrak{h}$-representation $S_{\mathfrak{k} / \mathfrak{h}} \otimes E_{\mu}$ lifts to a representation of the group $H$. Note that, since $\nu$ is dominant, $S_{\mathfrak{k} / \mathfrak{h}} \otimes E_{\mu+\nu}$ also can be lifted to an $H$-representation.

Proposition 2.5. The maps $b_{\mu}$ and $b_{\mu+\nu}$ fit in the following commutative diagram

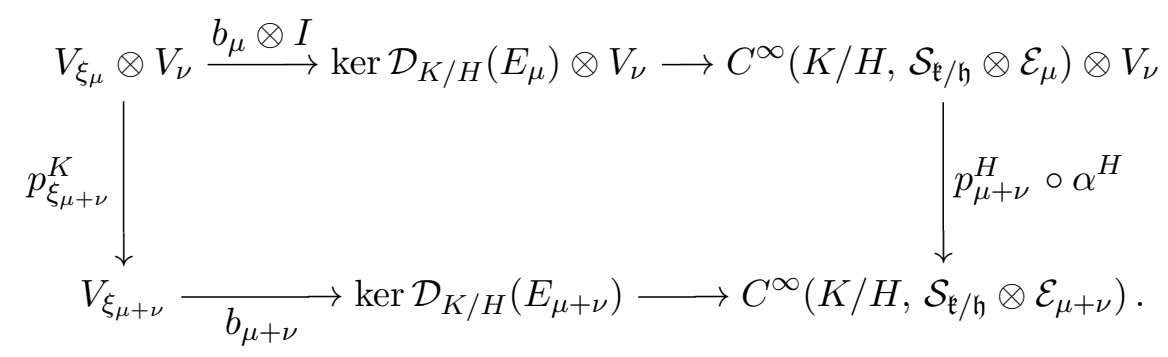

Proof. We need to prove the following diagram commutes

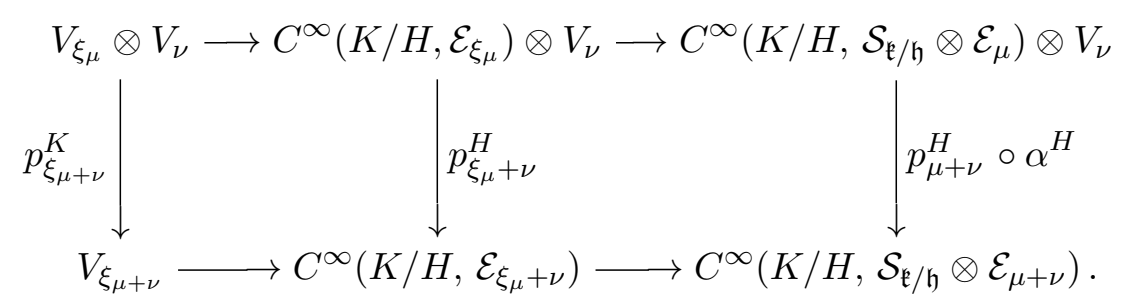


It is clear that the left square commutes. The commutativity of the right square amounts to prove that the following diagram is commutative:

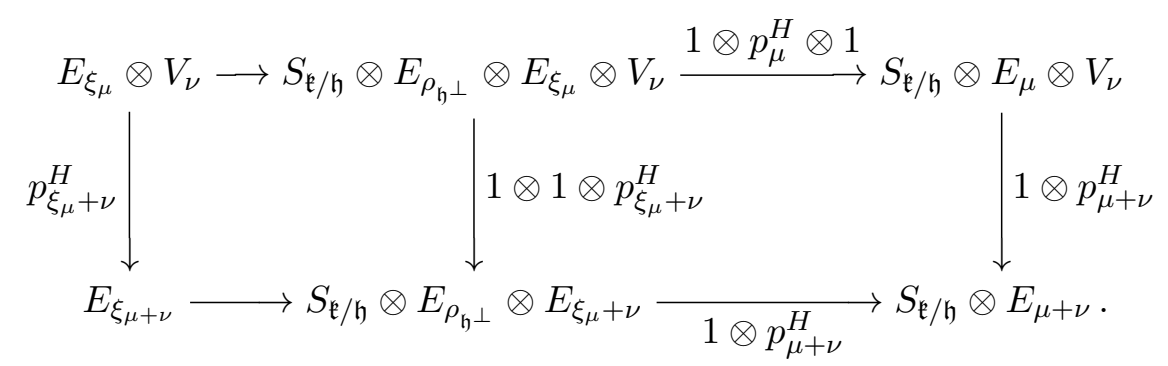

While the left part of the diagram clearly commutes, the commutativity of the right one follows from the associativity of the Cartan projection.

2.3. Highest weight submodule contribution. Some of the arguments in this subsection are adapted from [KW76]. We keep the notation of Section 1: $G$ is a connected reductive Lie group with complexified Lie algebra $\mathfrak{g}$ and Killing form $B$, and $H$ is a reductive subgroup of $G$ with complexified Lie algebra $\mathfrak{h}$ such that $B$ remains non degenerate on $\mathfrak{h}$. We fix a Cartan involution $\theta$ on $G$ and we assume that $H$ is $\theta$-stable. In order to state our result precisely we need to specify irreducible finite dimensional representations of $G$ and $H$ in terms of their highest weights. Let $\mathfrak{t}$ be a Cartan subalgebra of $\mathfrak{g}$. Such a Cartan subalgebra may and will be chosen so that it decomposes as $\mathfrak{t}=\mathfrak{t}_{\mathfrak{h}} \oplus \mathfrak{t}_{\mathfrak{h} \perp}$ where $\mathfrak{t}_{\mathfrak{h}}$ is a Cartan subalgebra of $\mathfrak{h}$. If $\nu$ is a linear form on $\mathfrak{t}$ it defines by restriction a linear form on $\mathfrak{t}_{\mathfrak{h}}$. In the other direction, when $\lambda$ is a linear form on $\mathfrak{t}_{\mathfrak{h}}$ we extend it to $\mathfrak{t}$ by letting it be 0 on $\mathfrak{t}_{\mathfrak{h} \perp}$.

First we choose a positive system $\Delta^{+}$for $\mathfrak{g}$ defined by a lexicographic order with $\mathfrak{t}_{\mathfrak{h}}$ first and then $\mathfrak{t}_{\mathfrak{h} \perp}$. When a positive system has been chosen we may construct as in [MZ14b] a positive system $\Delta^{+}(\mathfrak{h})$ for $\mathfrak{h}$ by

$$
\Delta^{+}(\mathfrak{h})=\left\{\left.\alpha\right|_{\mathfrak{t}_{\mathfrak{h}}} \in \Delta\left(\mathfrak{h}, \mathfrak{t}_{\mathfrak{h}}\right), \alpha \in \Delta^{+}, \mathfrak{g}^{(\alpha)} \subset \mathfrak{h},\left.\alpha\right|_{\mathfrak{t}_{\mathfrak{h}}} \neq 0\right\} .
$$

If $\lambda \in \mathfrak{t}_{\mathfrak{h}}^{*}$ is dominant for some positive system in $\Delta\left(\mathfrak{h}, \mathfrak{t}_{\mathfrak{h}}\right)$, we specify $\Delta^{+}$by choosing $\lambda$ as the first basis vector and define $\Delta^{+}(\mathfrak{h})$ as before. This makes $\lambda$ dominant for both $\Delta^{+}$and $\Delta^{+}(\mathfrak{h})$. Let $E_{\lambda}$ be an irreducible finite dimensional representation of $H$ with highest weight $\lambda$. Let $F_{\nu}$ be an irreducible representation of $G$ with highest weight $\nu$ with respect to $\Delta^{+}$. Then $\nu$ (restricted to $\mathfrak{t}_{\mathfrak{h}}$ ) is $\Delta^{+}(\mathfrak{h})$-dominant. As before, we get a surjective $H$-equivariant map

$$
E_{\lambda} \otimes F_{\nu} \stackrel{p_{\lambda+\nu}^{H}}{\longrightarrow} E_{\lambda+\nu}
$$

and a smooth $G$-equivariant map (denoted by the same symbol)

$$
C^{\infty}\left(G / H, \mathcal{S}_{\mathfrak{g} / \mathfrak{h}} \otimes \mathcal{E}_{\lambda} \otimes \mathcal{F}_{\nu}\right) \stackrel{p_{\lambda+\nu}^{H}}{\longrightarrow} C^{\infty}\left(G / H, \mathcal{S}_{\mathfrak{g} / \mathfrak{h}} \otimes \mathcal{E}_{\lambda+\nu}\right)
$$


We will use the isomorphism $\alpha^{H}$ and the projection $p_{\lambda+\nu}^{H}$ to relate the kernel of the Dirac operators with parameters $\lambda$ and $\lambda+\nu$ :

$$
C^{\infty}\left(G / H, \mathcal{S}_{\mathfrak{g} / \mathfrak{h}} \otimes \mathcal{E}_{\lambda}\right) \otimes F_{\nu} \stackrel{\alpha^{H}}{\longrightarrow} C^{\infty}\left(G / H, \mathcal{S}_{\mathfrak{g} / \mathfrak{h}} \otimes \mathcal{E}_{\lambda} \otimes \mathcal{F}_{\nu}\right) \stackrel{p_{\lambda+\nu}^{H}}{\longrightarrow} C^{\infty}\left(G / H, \mathcal{S}_{\mathfrak{g} / \mathfrak{h}} \otimes \mathcal{E}_{\lambda+\nu}\right)
$$

Recall that $\rho_{\mathfrak{g}}\left(\right.$ resp. $\left.\rho_{\mathfrak{h}}\right)$ is half the sum of positive roots in $\Delta^{+}\left(\right.$resp. $\left.\Delta^{+}(\mathfrak{h})\right)$ and that $\rho_{\mathfrak{h}^{\perp}}:=\rho_{\mathfrak{g}}-\rho_{\mathfrak{h}}$.

Proposition 2.6. The representation $S_{\mathfrak{g} / \mathfrak{h}} \otimes E_{\lambda}$ contains an irreducible subrepresentation $E_{\lambda+\rho_{\mathfrak{h} \perp}}$ with highest weight $\lambda+\rho_{\mathfrak{h} \perp}$.

Proof. The representation $E_{\lambda+\rho_{\mathfrak{h}} \perp}$ has infinitesimal character $\lambda+\rho_{\mathfrak{g}}$. Moreover the infinitesimal characters occurring in $S_{\mathfrak{g} / \mathfrak{h}} \otimes E_{\lambda}$ are of the form $\lambda+\rho_{\mathfrak{g}}-\Sigma(I)$ for $\Sigma(I):=\sum_{\alpha \in I} \alpha$ and $I \subset \Delta^{+}\left(\mathfrak{h}^{\perp}\right):=\Delta^{+} \cap \Delta\left(\mathfrak{h}^{\perp}\right)$. But $w\left(\lambda+\rho_{\mathfrak{g}}\right)=\lambda+\rho_{\mathfrak{g}}-\Sigma(I)$ implies that $w=1$ and $I$ is empty (see [KV95, Proposition 7.166]). So the $\mathcal{Z}(\mathfrak{h})$-primary component of $S_{\mathfrak{g} / \mathfrak{h}} \otimes E_{\lambda}$ with infinitesimal character $\lambda+\rho_{\mathfrak{g}}$ contains a representation $E_{\lambda+\rho_{\mathfrak{h}} \perp}$ with highest weight $\lambda+\rho_{\mathfrak{h} \perp}$.

Definition 2.7. Let

$$
\operatorname{ker}_{\text {top }} \mathcal{D}_{G / H}\left(E_{\lambda}\right):=\operatorname{ker} \mathcal{D}_{G / H}\left(E_{\lambda}\right) \cap C^{\infty}\left(G / H, \mathcal{E}_{\lambda+\rho_{\mathfrak{h}} \perp}\right) .
$$

Lemma 2.8. Let $f \in \operatorname{ker}_{\text {top }} \mathcal{D}_{G / H}\left(E_{\lambda}\right)$. Then for any $w \in F_{\nu}$, one has

$$
p_{\lambda+\nu}^{H} \circ \alpha^{H}(f \otimes w) \in \operatorname{ker} \mathcal{D}_{G / H}\left(E_{\lambda+\nu}\right) .
$$

The proof is based on the following lemma.

Lemma 2.9. Let $\mu$ be $a \Delta^{+}(\mathfrak{h})$-dominant analytically integral weight. If $f \in$ $C^{\infty}\left(G / H, \mathcal{E}_{\mu+\rho_{\mathfrak{h} \perp}}\right)$ then $\widehat{\mathcal{D}}_{G / H}\left(E_{\mu}\right) f$ has values in the subrepresentation of $S_{\mathfrak{g} / \mathfrak{h}} \otimes E_{\mu}$ which is contained in

$$
\oplus_{I} n_{I} E_{\mu+\rho_{\mathfrak{h}} \perp}-\Sigma(I)
$$

where $I$ runs over non empty subsets of $\Delta^{+}\left(\mathfrak{h}^{\perp}\right), \Sigma(I)=\sum_{\tau \in I} \tau$ and $n_{I}$ are non negative integers.

Proof. For any weight $\tau$ one has a decomposition $E_{\tau} \otimes \mathfrak{h}^{\perp}=\sum_{\beta \in \Delta\left(\mathfrak{h}^{\perp}\right)} n_{\beta} E_{\tau+\beta}$, for some $n_{\beta} \geq 0$, and corresponding projections

$$
p_{\tau}^{ \pm}: E_{\tau} \otimes \mathfrak{h}^{\perp} \longrightarrow \sum_{ \pm \beta>0} n_{\beta} E_{\tau+\beta} .
$$


When we take $\tau=\mu+\rho_{\mathfrak{h} \perp}$ we see that $\operatorname{Hom}_{H}\left(\operatorname{Im} p_{\mu+\rho_{\mathfrak{h} \perp}}^{+}, S_{\mathfrak{g} / \mathfrak{h}} \otimes E_{\mu}\right)=0$. In fact, the weight of $S_{\mathfrak{g} / \mathfrak{h}} \otimes E_{\mu}$ are of the form $\mu+\rho\left(\mathfrak{h}^{\perp}\right)-\Sigma(I)$ as in the proof of Proposition 2.6. So one has the following commutative diagram of $H$-equivariant linear maps.

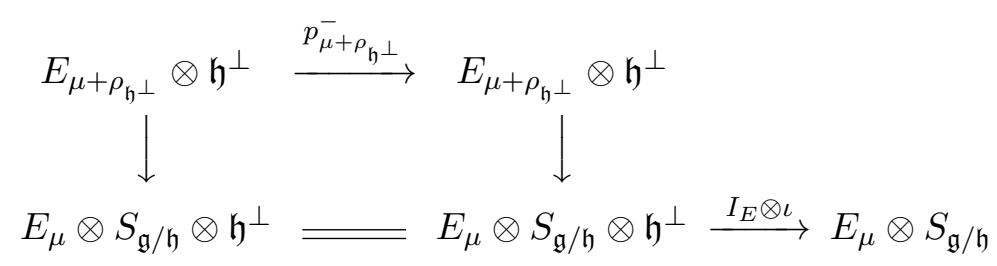

where $\iota$ is the contraction given by Clifford multiplication :

$$
\iota(v \otimes X)=\gamma(X) v \quad \forall v \in S_{\mathfrak{g} / \mathfrak{h}} \text { and } X \in \mathfrak{h}^{\perp} .
$$

Because of the presence of $p_{\mu+\rho_{\mathfrak{h}} \perp}^{-}$, this shows that the differential term $\widehat{\mathcal{D}}_{G / H}\left(E_{\mu}\right) f$ takes values in the subrepresentation of $S_{\mathfrak{g} / \mathfrak{h}} \otimes E_{\mu}$ which is contained in $\underset{I}{\oplus} n_{I} E_{\mu+\rho_{\mathfrak{h}} \perp}-\Sigma(I)$ where $I$ is non empty.

Proof for Lemma 2.8.

One has

$$
\mathcal{D}_{G / H}\left(E_{\lambda+\nu}\right) \circ p_{\lambda+\nu}^{H}=p_{\lambda+\nu}^{H} \circ \mathcal{D}_{G / H}\left(E_{\lambda} \otimes F_{\nu}\right)
$$

and

$$
\mathcal{D}_{G / H}\left(E_{\lambda} \otimes F_{\nu}\right) \circ \alpha^{H}=\alpha^{H} \circ\left(\mathcal{D}_{G / H}\left(E_{\lambda}\right) \otimes I_{F_{\nu}}\right)+A \circ \alpha^{H}
$$

where $A$ is the 0 th order invariant differential operator

$$
A:=-1 \otimes \widehat{D}_{F_{\nu}}\left(E_{\lambda}\right) \in 1 \otimes \operatorname{End}_{H}\left(S_{\mathfrak{g} / \mathfrak{h}} \otimes E_{\lambda} \otimes F_{\nu}\right) \subset\left(\mathcal{U}(\mathfrak{g}) \underset{\mathcal{U}(\mathfrak{h})}{\otimes} \operatorname{End}\left(S_{\mathfrak{g} / \mathfrak{h}} \otimes E_{\lambda} \otimes F_{\nu}\right)\right)^{H} .
$$

Now if $f$ is in the kernel of the Dirac operator $\mathcal{D}\left(E_{\lambda}\right)$, we get

$$
\mathcal{D}_{G / H}\left(E_{\lambda+\nu}\right) \circ p_{\lambda+\nu}^{H} \circ \alpha^{H}(f \otimes w)=p_{\lambda+\nu}^{H} \circ A \circ \alpha^{H}(f \otimes w) .
$$

By the assumption that $f$ takes its values in the particular subrepresentation $E_{\lambda+\rho_{\mathfrak{h}} \perp}$, one has that $p_{\lambda+\nu}^{H} \circ \alpha^{H}(f \otimes w)$ and $p_{\lambda+\nu}^{H} \circ A \circ \alpha^{H}(f \otimes w)$ both are in a subbundle $E_{\lambda+\nu+\rho_{\mathfrak{h}} \perp}$ with highest weight $\lambda+\nu+\rho_{\mathfrak{h} \perp}$. Applying Lemma 2.9 with $\mu=\lambda+\nu$ we get that the term $\widehat{\mathcal{D}}_{G / H}\left(E_{\lambda+\nu}\right) \circ$ $p_{\lambda+\nu}^{H} \circ \alpha^{H}(f \otimes w)$ has values in a subrepresentation of the form $\sum_{I} n_{I} E_{\lambda+\nu+\rho_{\mathfrak{h} \perp}-\Sigma(I)}$.

Next we show that the cubic term also takes values in a subbundle of the same form. Since $\mathcal{D}_{G / H}\left(E_{\lambda}\right) f=0$ and $f$ satisfies the assumption of Lemma 2.9 with $\mu=\lambda$, it follows that

$$
\left(1 \otimes \gamma(\mathbf{c}) \otimes I_{E_{\lambda}}\right) f=-\widehat{\mathcal{D}}_{G / H}\left(E_{\lambda}\right) f \in C^{\infty}\left(G / H, \oplus_{I} \mathcal{E}_{\lambda+\rho_{\mathfrak{h}} \perp-\Sigma(I)}\right) .
$$


This implies that

$$
\begin{aligned}
\left(1 \otimes \gamma(\mathbf{c}) \otimes I_{E_{\lambda+\nu}}\right)\left(p_{\lambda+\nu}^{H} \circ \alpha^{H}(f \otimes w)\right) & \\
& \left.=p_{\lambda+\nu}^{H} \circ \alpha^{H}\left(1 \otimes \gamma(\mathbf{c}) \otimes I_{E_{\lambda}}\right) f \otimes w\right) \notin C^{\infty}\left(G / H, \mathcal{E}_{\lambda+\nu+\rho_{\mathfrak{h}}}\right) .
\end{aligned}
$$

We then see that the cubic term of $\mathcal{D}_{G / H}\left(E_{\lambda+\nu}\right)$ also has values on $\alpha^{H}(f \otimes w)$ in $\sum_{I} n_{I} E_{\lambda+\nu+\rho_{\mathfrak{h} \perp}-\Sigma(I)}$. It follows that the left hand side of formula (12) takes values in a subbundle of the form $\sum_{I} n_{I} E_{\lambda+\nu+\rho_{\mathfrak{h} \perp}-\Sigma(I)}$ while the right hand side is a section of a subbundle $E_{\lambda+\nu+\rho_{\mathfrak{h} \perp}}$ with highest weight $\lambda+\nu+\rho_{\mathfrak{h} \perp}$. Hence both terms must vanish.

\section{The Fundamental SERIES}

We now show that the embedding of a globalization of the fundamental series in the kernel of the Dirac operator, as defined by Mehdi and Zierau in [MZ14b], commutes with the Zuckerman translation functor. Let us give a precise statement.

In this section we assume that $H$ is a compact, connected and $\theta$-stable Lie subgroup of $G$. So we may assume that $H \subset K$. At the level of Lie algebras, we have

$$
\mathfrak{g}=\mathfrak{h}+\mathfrak{h}^{\perp}=\mathfrak{h}+\left(\mathfrak{h}^{\perp} \cap \mathfrak{k}\right)+\left(\mathfrak{h}^{\perp} \cap \mathfrak{s}\right)
$$

where $\mathfrak{g}=\mathfrak{k} \oplus \mathfrak{s}$ is the Cartan decomposition associated with $\theta$. The Cartan subalgebra $\mathfrak{t}=\mathfrak{t}_{\mathfrak{h}}+\mathfrak{t}_{\mathfrak{h} \perp}$ of $\mathfrak{g}$ is chosen so that $\mathfrak{t}_{\mathfrak{h} \perp}=\left(\mathfrak{t}_{\mathfrak{h} \perp} \cap \mathfrak{k}\right)+\mathfrak{a}$ where $\mathfrak{a}$ is an abelian subspace of $\mathfrak{s}$, $\mathfrak{t}_{\mathfrak{k}}=\mathfrak{t}_{\mathfrak{h}}+\left(\mathfrak{t}_{\mathfrak{h}} \perp \cap \mathfrak{k}\right)$ is a Cartan subalgebra of $\mathfrak{k}$ and $\mathfrak{t}=\mathfrak{t}_{\mathfrak{k}}+\mathfrak{a}$ is a maximally compact Cartan subalgebra of $\mathfrak{g}$.

We now choose positive systems as in [MZ14b]. Let $\Delta^{+} \subset \Delta(\mathfrak{t}+\mathfrak{a}, \mathfrak{g})$ be defined by a lexicographic order with $\mathfrak{t}_{\mathfrak{h}}$ first, then $\mathfrak{t}_{\mathfrak{h}} \perp$, and $\mathfrak{a}$. Such a positive system has the property that

$$
\Delta^{+}(\mathfrak{h}):=\left\{\left.\alpha\right|_{\mathfrak{t}_{\mathfrak{h}}}: \mathfrak{g}^{(\alpha)} \subset \mathfrak{h}, \alpha \in \Delta^{+} \text {and } \alpha_{\mathfrak{t}_{\mathfrak{h}}} \neq 0\right\}
$$

is a positive system of roots in $\mathfrak{h}$. Here we are denoting the $\alpha$-root space in $\mathfrak{g}$ by $\mathfrak{g}^{(\alpha)}$. A Borel subalgebra of $\mathfrak{g}$ is associated to $\Delta^{+}$in the usual way by

$$
\mathfrak{b}=\mathfrak{t}+\mathfrak{u}, \quad \mathfrak{u}=\sum_{\alpha \in \Delta^{+}} \mathfrak{g}^{(\alpha)}
$$

Let $E_{\mu}$ be an irreducible representation of $\mathfrak{h}$ with highest weight $\mu \in \mathfrak{t}_{\mathfrak{h}}^{*}$ which is $\Delta^{+}(\mathfrak{t}, \mathfrak{g})$ dominant. As before we assume that the tensor product $S_{\mathfrak{g} / \mathfrak{h}} \otimes E_{\mu}$ lifts to a representation of the group $H$. We define $\xi_{\mu} \in \mathfrak{t}_{\mathfrak{k}}^{*}$ as

$$
\xi_{\mu}=\mu-\left(\rho_{\mathfrak{k}}-\rho_{\mathfrak{h}}\right)
$$


and choose $\lambda_{\mu} \in \mathfrak{t}^{*}$ such that

$$
\left.\lambda_{\mu}\right|_{\mathfrak{t}_{\mathfrak{k}}}=\xi_{\mu}-\rho_{\mathfrak{s}}=\mu-\rho_{\mathfrak{k}}+\rho_{\mathfrak{h}}-\rho_{\mathfrak{s}} \quad \text { and }\left.\quad \lambda_{\mu}\right|_{\mathfrak{a}}=0
$$

where $\rho_{\mathfrak{s}}:=\rho_{\mathfrak{g}}-\rho_{\mathfrak{k}}$. Let $V_{\xi_{\mu}}$ be the irreducible representation of $K$ with highest weight $\xi_{\mu}$, and assume that $S_{\mathfrak{g} / \mathfrak{k}} \otimes V_{\xi_{\mu}}$ lifts to a representation of $K$ (see Section 1). Let $A_{\mathfrak{b}}^{\infty}\left(\lambda_{\mu}\right)$ be a smooth globalization with moderate growth of the fundamental series representation $A_{\mathfrak{b}}\left(\lambda_{\mu}\right)$, that is the cohomologically induced $(\mathfrak{g}, K)$-module with parameter $\left(\mathfrak{b}, \lambda_{\mu}\right)$ ([KV95, Chapter $5]$ ). Since $A_{\mathfrak{b}}\left(\lambda_{\mu}\right)$ is an admissible $(\mathfrak{g}, K)$-module, such a globalization exists and is unique up to isomorphism by Casselman-Wallach globalization Theorem [Wal92, Chapter 11].

\subsection{First step.}

Theorem 3.1. (Corollary 1.14, Lemma 1.20 (case $H=K$ ) in [MZ14b])

There exists a one-to-one G-homomorphism

$$
A_{\mathfrak{b}}^{\infty}\left(\lambda_{\mu}\right) \stackrel{A_{\mu}}{\longrightarrow} \operatorname{ker} \mathcal{D}_{G / K}\left(V_{\xi_{\mu}}\right) .
$$

Moreover for any possible choice of the map $A_{\mu}$, the range of $A_{\mu}$ is contained in the space of sections of the fiber bundle associated to the isotypic component of $S_{\mathfrak{g} / \mathfrak{k}} \otimes V_{\xi_{\mu}}$ with highest weight $\xi_{\mu}+\rho_{\mathfrak{s}}$.

Let $F_{\nu}$ be an irreducible finite dimensional representation of $G$ with highest weight $\nu$ such that $\left.\nu\right|_{\mathfrak{a}}=0$.

Lemma 3.2. There exists a unique (up to scalar) $G$-intertwining map of smooth $G$-modules

$$
\iota: A_{\mathfrak{b}}^{\infty}\left(\lambda_{\mu+\nu}\right) \rightarrow A_{\mathfrak{b}}^{\infty}\left(\lambda_{\mu}\right) \otimes F_{\nu} .
$$

Proof. The representation $A_{\mathfrak{b}}^{\infty}\left(\lambda_{\mu}\right) \otimes F_{\nu}$ is a smooth $G$-representation of moderate growth because $A_{\mathfrak{b}}^{\infty}\left(\lambda_{\mu}\right)$ is of moderate growth and $F_{\nu}$ is finite dimensional. Moreover the space of $K$ finite vectors of $A_{\mathfrak{b}}^{\infty}\left(\lambda_{\mu}\right) \otimes F_{\nu}$ is $A_{\mathfrak{b}}\left(\lambda_{\mu}\right) \otimes F_{\nu}$. The $\mathcal{Z}(\mathfrak{g})$-primary component $\tilde{p}_{\lambda_{\mu}+\nu+\rho_{\mathfrak{g}}}\left(A_{\mathfrak{b}}^{\infty}\left(\lambda_{\mu}\right) \otimes\right.$ $F_{\nu}$ ), with generalized infinitesimal character $\lambda_{\mu}+\nu+\rho_{\mathfrak{g}}$, is then a smooth $G$-representation of moderate growth as well, and its space of $K$-finite vectors is the $\mathcal{Z}(\mathfrak{g})$-primary component of $A_{\mathfrak{b}}\left(\lambda_{\mu}\right) \otimes F_{\nu}$ with generalized infinitesimal character $\lambda_{\mu}+\nu+\rho_{\mathfrak{g}}$. By [KV95, Theorem 7.237] the latter is isomorphic to $A_{\mathfrak{b}}\left(\lambda_{\mu}+\nu\right)$. In particular, the irreducible representation $A_{\mathfrak{b}}\left(\lambda_{\mu}+\nu\right)$ occurs with multiplicity one in the tensor product $A_{\mathfrak{b}}\left(\lambda_{\mu}\right) \otimes F_{\nu}$. Finally, again by Casselman-Wallach globalization Theorem [Wal92, Chapter 11], one has

$$
\tilde{p}_{\lambda_{\mu}+\nu+\rho_{\mathfrak{g}}}\left(A_{\mathfrak{b}}^{\infty}\left(\lambda_{\mu}\right) \otimes F_{\nu}\right) \simeq A_{\mathfrak{b}}^{\infty}\left(\lambda_{\mu}+\nu\right)
$$


with $\lambda_{\mu+\nu}=\lambda_{\mu}+\nu$. The existence of the map $\iota: A_{\mathfrak{b}}^{\infty}\left(\lambda_{\mu+\nu}\right) \rightarrow A_{\mathfrak{b}}^{\infty}\left(\lambda_{\mu}\right) \otimes F_{\nu}$ now follows. Its unicity relies on the fact that $A_{\mathfrak{b}}\left(\lambda_{\mu}+\nu\right)$ has multiplicity one in $A_{\mathfrak{b}}\left(\lambda_{\mu}\right) \otimes F_{\nu}$.

From Theorem 3.1 we know that this embedding $\iota$ takes value in the highest weight subbundle of $S_{\mathfrak{g} / \mathfrak{k}} \otimes V_{\xi}$, that is $\operatorname{ker}_{\text {top }} \mathcal{D}_{G / K}\left(V_{\xi_{\mu}}\right)$. In particular, $p_{\xi_{\mu}+\left.\nu\right|_{\mathfrak{t}_{\mathfrak{k}}} ^{K}}^{K} \alpha^{K}$ applies the image of $\left(A_{\mu} \otimes I\right) \circ \iota$ to the kernel of the Dirac operator by Lemma 2.8. Here $\alpha^{K}$ is defined in a similar way as for $\alpha^{H}$ in Proposition 2.1. Since $\xi_{\mu}+\nu=\xi_{\mu+\nu}$, this allows to define

$$
A_{\mu+\nu}=p_{\xi_{\mu+\nu}}^{K} \circ \alpha^{K} \circ\left(A_{\mu} \otimes I\right) \circ \iota .
$$

Proposition 3.3. The following diagram is commutative

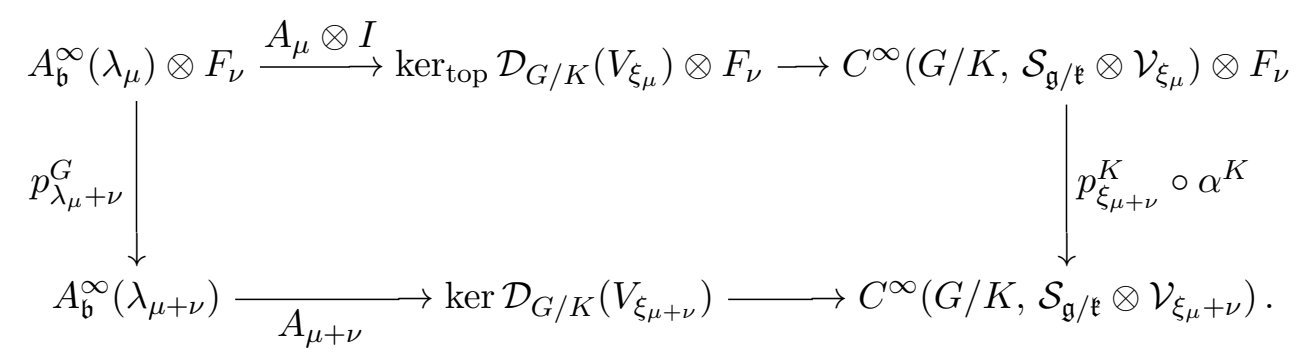

Proof. The infinitesimal character of $A_{\mathfrak{b}}^{\infty}\left(\lambda_{\mu}\right)$ is $\lambda_{\mu}+\rho_{\mathfrak{g}}$. So the $\mathcal{Z}(\mathfrak{g})$-primary components of $A_{\mathfrak{b}}^{\infty}\left(\lambda_{\mu}\right) \otimes F_{\nu}$ have generalized infinitesimal character of the form $\lambda_{\mu}+\rho_{\mathfrak{g}}+\nu^{\prime}$ where $\nu^{\prime}$ is a weight of $F_{\nu}$. On the corresponding component the Casimir operator $\Omega_{G}$ acts by

$$
\Omega_{G}=\left\|\lambda_{\mu}+\rho_{\mathfrak{g}}+\nu^{\prime}\right\|^{2}-\left\|\rho_{\mathfrak{g}}\right\|^{2} .
$$

On the other hand, on the space $\operatorname{ker} \mathcal{D}_{G / K}\left(V_{\xi_{\mu+\nu}}\right)$ the Kostant-Parthasarathy formula implies that the Casimir operator acts by

$$
\Omega_{G}=\left\|\xi_{\mu+\nu}+\rho_{\mathfrak{k}}\right\|^{2}-\left\|\rho_{\mathfrak{g}}\right\|^{2} .
$$

So if $p_{\xi_{\mu+\nu}}^{K} \circ \alpha^{K} \neq 0$ on the component of $\left(A_{\mu} \otimes I\right)\left(A_{\mathfrak{b}}\left(\lambda_{\mu}\right) \otimes F_{\nu}\right)$ with generalized infinitesimal character $\lambda_{\mu}+\rho_{\mathfrak{g}}+\nu^{\prime}$ we should have

$$
\left\|\xi_{\mu+\nu}+\rho_{\mathfrak{k}}\right\|^{2}=\left\|\lambda_{\mu}+\rho_{\mathfrak{g}}+\nu^{\prime}\right\|^{2}
$$

But as $\xi_{\mu+\nu}+\rho_{\mathfrak{k}}=\lambda_{\mu}+\rho_{\mathfrak{g}}+\nu$, this implies that

$$
\lambda_{\mu}+\rho_{\mathfrak{g}}+\nu^{\prime}=w\left(\lambda_{\mu}+\rho_{\mathfrak{g}}+\nu\right)
$$

for some element $w$ in the Weyl group of $\mathfrak{g}$. By a standard argument this is possible only if $\nu^{\prime}=\nu$ (see $\left[\mathrm{KV} 95\right.$, Proposition 7.166]). In conclusion, $p_{\xi_{\mu}+\nu}^{K} \circ \alpha^{K} \circ\left(A_{\mu} \otimes 1\right)$ vanishes on the kernel of $p_{\lambda_{\mu}+\nu}^{G}$. 
3.2. Second step. We review the one-to-one $G$-maps constructed by Mehdi and Zierau in [MZ14b]

$$
\operatorname{ker} \mathcal{D}_{G / K}\left(V_{\xi_{\mu}}\right) \stackrel{B_{\mu}}{\longrightarrow} \operatorname{ker} \mathcal{D}_{G / H}\left(E_{\mu}\right)
$$

and we prove that these maps are compatible with translation functors. These maps depend on the embedding defined in (10)

$$
b_{\mu}: V_{\xi_{\mu}} \rightarrow \operatorname{ker} \mathcal{D}_{K / H}\left(E_{\mu}\right)
$$

More precisely the construction goes as follows. First, we have an isomorphism

$$
\phi_{\mu}: C^{\infty}\left(G / H, \mathcal{S}_{\mathfrak{g} / \mathfrak{h}} \otimes \mathcal{E}_{\mu}\right) \rightarrow C^{\infty}\left(G / K, \mathcal{S}_{\mathfrak{g} / \mathfrak{k}} \otimes C^{\infty}\left(K / H, \mathcal{S}_{\mathfrak{k} / \mathfrak{h}} \otimes \mathcal{E}_{\mu}\right)\right),
$$

and under this isomorphism the Dirac operator writes :

$$
\phi_{\mu}\left(\mathcal{D}_{G / H}\left(E_{\mu}\right) f\right)=\mathcal{D}_{G / K}\left(C^{\infty}\left(K / H, \mathcal{S}_{\mathfrak{k} / \mathfrak{h}} \otimes \mathcal{E}_{\mu}\right)\right) \phi(f)+1 \otimes \mathcal{D}_{K / H}\left(E_{\mu}\right) f
$$

Now let $b_{\mu}: V_{\xi_{\mu}} \rightarrow \operatorname{ker} \mathcal{D}_{K / H}\left(E_{\mu}\right)$ be a $K$-homomorphism. The map $B_{\mu}$ is then defined by

$$
\begin{aligned}
B_{\mu}: C^{\infty}\left(G / K, \mathcal{S}_{\mathfrak{g} / \mathfrak{k}} \otimes \mathcal{V}_{\xi_{\mu}}\right) & \rightarrow C^{\infty}\left(G / H, \mathcal{S}_{\mathfrak{g} / \mathfrak{h}} \otimes \mathcal{E}_{\mu}\right) \\
f & \mapsto \phi_{\mu}^{-1}\left(g \mapsto b_{\mu}(f(g))\right),
\end{aligned}
$$

and if $f \in \operatorname{ker} \mathcal{D}_{G / K}\left(V_{\xi_{\mu}}\right)$ then $B_{\mu}(f)$ is in the kernel of $D_{G / H}\left(E_{\mu}\right)$. Recall that for $\beta \in \mathfrak{t}^{*}$, the map $p_{\beta}^{K}$ (resp. $p_{\beta}^{H}$ ) denotes the projection onto the irreducible $K$-isotypic (resp. $H$-isotypic) component of highest weight $\beta$.

Proposition 3.4. For all $\mu$ and $\nu$ in the same Weyl chamber for $\mathfrak{g}$, the following diagram is commutative.

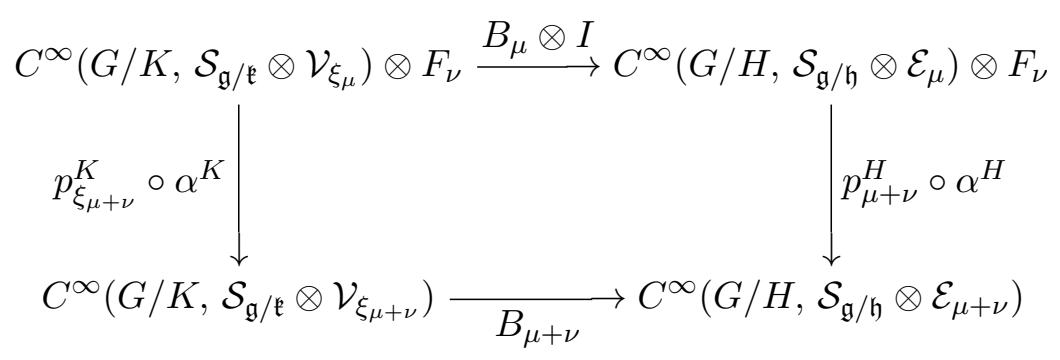

Proof. By construction the map $B_{\mu}$ is given by the map $\phi_{\mu} \circ\left(I \otimes b_{\mu}\right)$ where $\phi_{\mu}$ is the isomorphism of equation (15) and

$$
I \otimes b_{\mu}: C^{\infty}\left(G / K, \mathcal{S}_{\mathfrak{g} / \mathfrak{k}} \otimes \mathcal{V}_{\xi_{\mu}}\right) \rightarrow C^{\infty}\left(G / K, \mathcal{S}_{\mathfrak{g} / \mathfrak{k}} \otimes \mathcal{C}^{\infty}\left(K / H, \mathcal{S}_{\mathfrak{k} / \mathfrak{h}} \otimes \mathcal{E}_{\mu}\right)\right)
$$


defined by $I \otimes b_{\mu}(f)(g)=I \otimes b_{\mu}(f(g))$. This yields the following sequence of commutative diagrams.

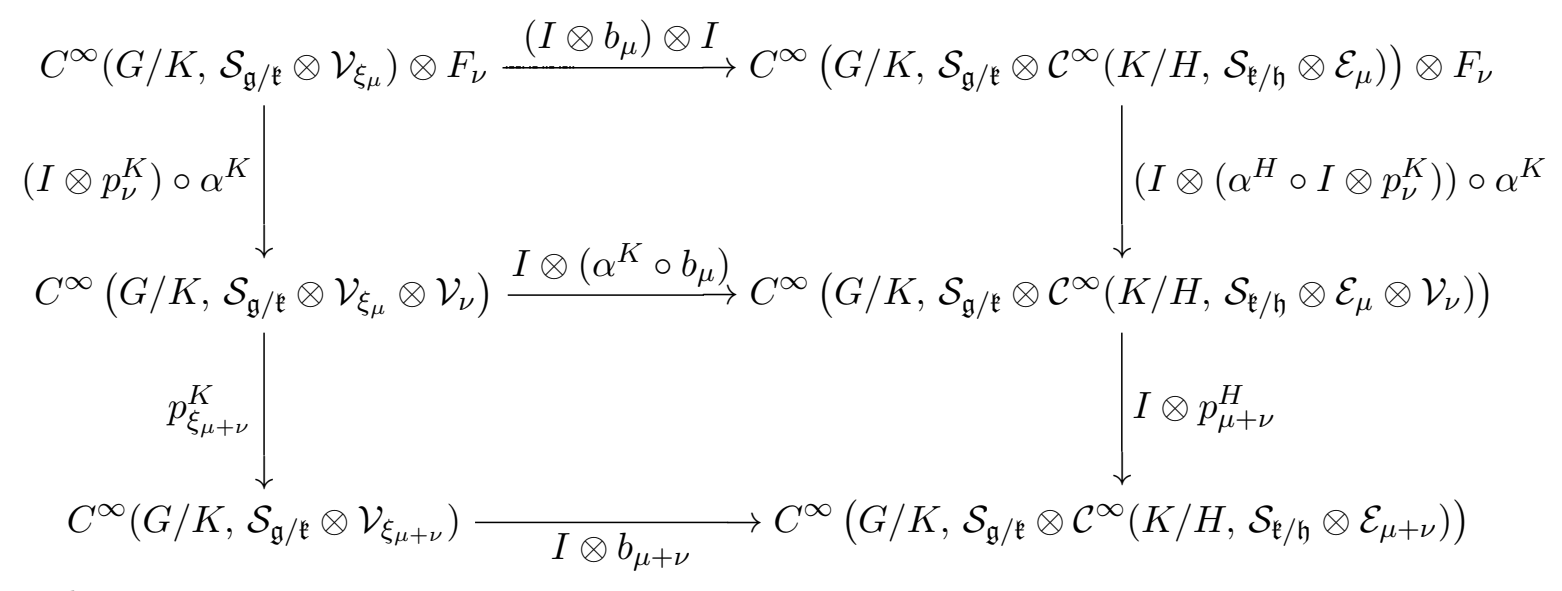

and

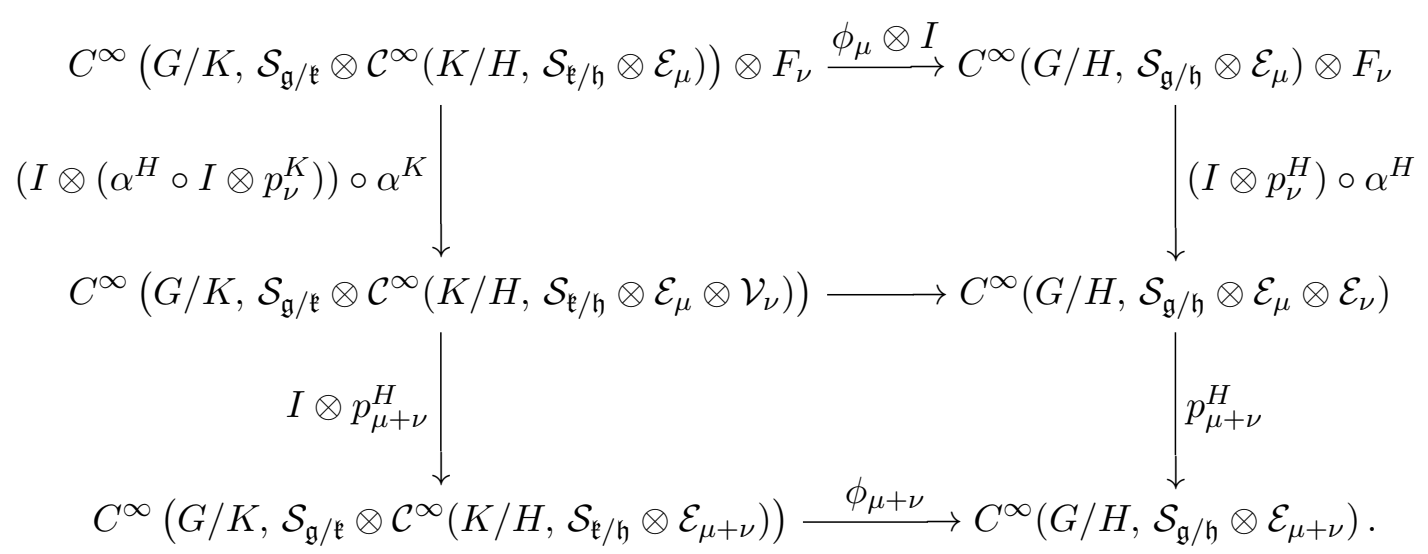

3.3. Conclusion. Steps 1 and 2 above can be summarized as follows. Let $e_{e}$ be the evaluation at the identity. It follows from [MZ14b, Section 1.3.3] that for all $\mu$ the evaluation at the identity of a section in the range of the map $B_{\mu} \circ A_{\mu}$ has values in the $H$-submodule $V_{0}(\mu)$ of $E_{\mu} \otimes S_{\mathfrak{g} / \mathfrak{h}}$ defined as its isotypic component of highest weight $\mu+\rho_{\mathfrak{h} \perp}-2 \rho_{\mathfrak{h} \perp} \cap \mathfrak{k}$.

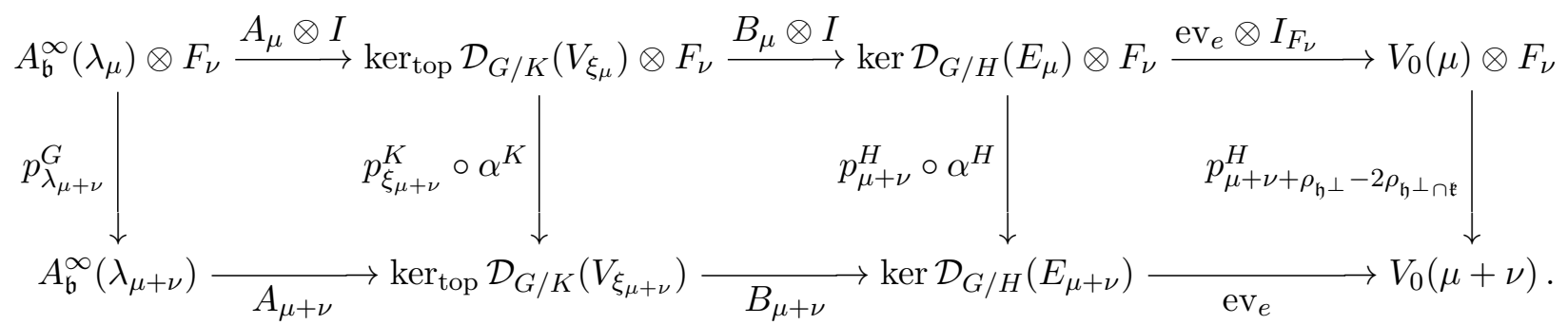

In the next section we will simply drop $A_{\mu}$ and $B_{\mu}$ from the notations. Moreover, the group named here $G$ will be replaced by a subgroup $M$ (of a parabolic subgroup) of $G$ and 
the group $H$ will be replaced by $H \cap M$. So we will simply write without further reference to this section that

$$
\left(p_{\lambda_{\mu+\nu}}^{M}(f \otimes w)\right)(e)=p_{\mu+\nu+\rho_{\mathfrak{m} \cap \mathfrak{h}^{\perp}}-2 \rho_{\mathfrak{m} \cap \mathfrak{k} \cap \mathfrak{h}^{\perp}}}^{H \cap M}(f(e) \otimes w)
$$

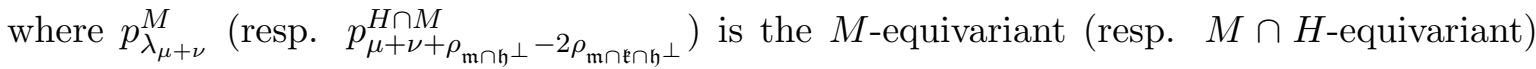
defined as in (11).

\section{THE PRINCIPAL SERIES}

We start this section with some notations from [MZ14b]. We choose a Cartan subalgebra of $\mathfrak{g}$ as follows. Fix a maximal abelian subspace $\mathfrak{a}_{\mathfrak{h}}$ of $\mathfrak{h} \cap \mathfrak{s}$ and let $\mathfrak{l}:=\mathfrak{z}_{\mathfrak{g}}\left(\mathfrak{a}_{\mathfrak{h}}\right)$ be the centralizer of $\mathfrak{a}_{\mathfrak{h}}$ in $\mathfrak{g}$. Fix a Cartan subalgebra $\mathfrak{t}_{\mathfrak{h}}$ of $\mathfrak{h} \cap \mathfrak{k} \cap \mathfrak{l}$ and extend $\mathfrak{t}_{\mathfrak{h}}$ to a Cartan subalgebra $\mathfrak{t}=\mathfrak{t}_{\mathfrak{h}}+\mathfrak{t}_{\mathfrak{h} \perp}$ of $\mathfrak{k} \cap \mathfrak{l}$ with $\mathfrak{t}_{\mathfrak{h} \perp} \subset \mathfrak{h}^{\perp} \cap \mathfrak{k} \cap \mathfrak{l}$. Then choose $\mathfrak{a}_{\mathfrak{h} \perp} \subset \mathfrak{h}^{\perp} \cap \mathfrak{s} \cap \mathfrak{l}$ so that $\mathfrak{a}_{\mathfrak{h}}+\mathfrak{t}+\mathfrak{a}_{\mathfrak{h} \perp}$ is a Cartan subalgebra of $\mathfrak{l}$. Letting $\mathfrak{a}:=\mathfrak{a}_{\mathfrak{h}}+\mathfrak{a}_{\mathfrak{h}}$, we see that $\mathfrak{a}+\mathfrak{t}$ is also a Cartan subalgebra of $\mathfrak{g}$.

Let $\Delta:=\Delta(\mathfrak{a}+\mathfrak{t}, \mathfrak{g})$ be the set of $\mathfrak{a}+\mathfrak{t}$-roots in $\mathfrak{g}$. Fix a positive system $\Delta^{+}$in $\Delta$ by a lexicographic order with a basis of $\mathfrak{a}_{\mathfrak{h}}$ first, then (in order) bases of $\mathfrak{t}_{\mathfrak{h}}, \mathfrak{t}_{\mathfrak{h} \perp}$ and $\mathfrak{a}_{\mathfrak{h}}{ }^{\perp}$. A positive system $\Delta^{+}(\mathfrak{h})$ of $\left(\mathfrak{a}_{\mathfrak{h}}+\mathfrak{t}_{\mathfrak{h}}\right)$-roots in $\mathfrak{h}$ is chosen using the lexicographic order with the same basis of $\mathfrak{a}_{\mathfrak{h}}$ as above, followed by the basis of $\mathfrak{t}_{\mathfrak{h}}$.

Having fixed a positive system of roots $\Delta^{+}$in $\mathfrak{g}$, we may define a parabolic subalgebra of $\mathfrak{g}$ as follows. Set

$$
\Sigma^{+}:=\left\{\alpha \in \Delta^{+}:\left.\alpha\right|_{\mathfrak{a}_{\mathfrak{h}}} \neq 0\right\} .
$$

Then $\mathfrak{p}:=\mathfrak{l}+\mathfrak{n}$ is a parabolic subalgebra of $\mathfrak{g}$ where

$$
\mathfrak{n}:=\sum_{\alpha \in \Sigma^{+}} \mathfrak{g}^{(\alpha)} .
$$

By the above choice of $\Delta^{+}, \mathfrak{p}$ is the complexification of a (real) subalgebra of $\operatorname{Lie}(G)$. We let $P$ be the connected subgroup of $G$ corresponding to this real parabolic Lie algebra. Then $P$ is a parabolic subgroup of $G$ such that $P \cap H$ is a minimal parabolic subgroup of $H$ (see Section 3.2 in [MZ14b]). It will be convenient for us to write $\mathfrak{l}=\mathfrak{m}+\mathfrak{a}_{\mathfrak{h}}$ with

$$
\mathfrak{m}=\sum_{\alpha \in \Delta,\left.\alpha\right|_{\mathfrak{a}_{\mathfrak{h}}=0}} \mathfrak{g}^{(\alpha)}+\left(\mathfrak{a}_{\mathfrak{h}} \perp+\mathfrak{t}\right) .
$$

Therefore, one has the decomposition $\mathfrak{p}=\mathfrak{m}+\mathfrak{a}_{\mathfrak{h}}+\mathfrak{n}$ of $\mathfrak{p}$ and the corresponding decomposition of $P$, which we write as $P=M A_{\mathfrak{h}} N$.

For the construction of the intertwining map, it is necessary to assume that the subgroup $H$ is not too small for certain Dirac cohomology spaces to be non zero. This is ensured by 
the following assumption (Assumption 1.24 in [MZ14b]):

there is no $\operatorname{root} \beta \in \Delta\left(\mathfrak{t}_{\mathfrak{h}}+\mathfrak{t}_{\mathfrak{h} \perp}+\mathfrak{a}_{\mathfrak{h} \perp}, \mathfrak{m}\right)$ so that $\left.\beta\right|_{\mathfrak{t}_{\mathfrak{h}}}=0$.

Now consider a highest weight representation $E_{\mu}$ of $H$ with highest weight $\mu$ such that the tensor product representation $S_{\mathfrak{g} / \mathfrak{h}} \otimes E_{\mu}$ of $\mathfrak{h}$ lifts to a representation of $H$, and where $\mu \in\left(\mathfrak{a}_{\mathfrak{h}}+\mathfrak{t}_{\mathfrak{h}}\right)^{*}$ is a $\Delta^{+}(\mathfrak{h})$-dominant integral weight satisfying the following conditions:

(i) $\left\langle\mu+\rho_{\mathfrak{m} \cap \mathfrak{h}}-\Sigma(I), \beta\right\rangle \geq 0$, for all $\beta \in \Delta^{+}(\mathfrak{m} \cap \mathfrak{h})$ and $I \subset \Delta^{+}\left(\mathfrak{m} \cap \mathfrak{h}^{\perp}\right)$,

(ii) $\left\langle\mu+\rho_{\mathfrak{m} \cap \mathfrak{h}}-2 \rho_{\mathfrak{m} \cap \mathfrak{k} \cap \mathfrak{h}^{\perp}}, \beta\right\rangle>0$, for all $\beta \in \Delta^{+}(\mathfrak{m} \cap \mathfrak{h})$

where $\Sigma(I)$ is the sum of the elements in $I$ (see Section 2.3). Under these assumptions, $E_{\widetilde{\mu}}:=\left(E_{\mu}\right)^{\mathfrak{n} \cap \mathfrak{h}}$ is an irreducible representation of $\mathfrak{m} \cap \mathfrak{h}$ of highest weight $\left.\mu\right|_{\mathfrak{t}_{\mathfrak{h}}}$ and $S_{\mathfrak{m} / \mathfrak{m} \cap \mathfrak{h}} \otimes$ $E_{\widetilde{\mu}}$ contains the irreducible highest weight representation of $\mathfrak{m} \cap \mathfrak{h}$ having highest weight $\mu+\rho_{\mathfrak{m} \cap \mathfrak{h}^{\perp}}-2 \rho_{\mathfrak{m} \cap \mathfrak{k} \cap \mathfrak{h}^{\perp}}$ (Section 1.3.3 in [MZ14b]). Moreover, by Theorem 3.8 in [MZ14b], there exist a representation $\widetilde{W}_{\mu}$ of $P$ and a non-zero $G$-intertwining map

$$
\mathcal{P}_{\mathcal{E}_{\mu}}: C^{\infty}\left(G / P, \widetilde{\mathcal{W}}_{\mu}\right) \rightarrow C^{\infty}\left(G / H, \mathcal{E}_{\mu} \otimes \mathcal{S}\right)
$$

such that $\operatorname{Im}\left(\mathcal{P}_{\mathcal{E}_{\mu}}\right) \subset \operatorname{ker}\left(\mathcal{D}_{G / H}\left(E_{\mu}\right)\right)$. Both the $P$-representation $\widetilde{W}_{\mu}$ and the intertwining map $\mathcal{P}_{\mathcal{E}_{\mu}}$ are explicitly described. The representation $\widetilde{W}_{\mu}$ is constructed as follows. Let $W_{\lambda_{\mu}}$ be a smooth globalization of the cohomologically induced representation

$$
W_{\lambda_{\mu}}=A_{\mathfrak{b} \cap \mathfrak{m}}^{\infty}\left(\lambda_{\mu}\right)
$$

given along with an equivariant map of $M$-modules into $\operatorname{ker}\left(D_{M / M \cap H}\left(E_{\widetilde{\mu}}\right)\right)$ as in Section 3 . Then the representation $W_{\mu}$ of $P$ is then

$$
\widetilde{W}_{\mu}:=W_{\lambda_{\mu}} \otimes \mathbb{C}_{-\left.2 \rho_{\mathfrak{h}}\right|_{\mathfrak{a} \mathfrak{h}}} .
$$

Recall from [MZ14b, Lemma 1.25] that there is an embedding of spinors

$$
S_{\mathfrak{m} / \mathfrak{m} \cap \mathfrak{h}} \hookrightarrow S_{\mathfrak{g} / \mathfrak{h}}
$$

The intertwining map $\mathcal{P}_{\mathcal{E}_{\mu}}$ is defined by

$$
\left(\mathcal{P}_{\mathcal{E}_{\mu}} \varphi\right)(g)=\int_{H \cap K} \ell \cdot t_{\mu}(\varphi(g \ell)) d \ell
$$

for a non-zero $t_{\mu} \in \operatorname{Hom}_{P \cap H}\left(W_{\lambda_{\mu}} \otimes \mathbf{C}_{-\left.2 \rho_{\mathfrak{h}}\right|_{\mathfrak{h}}}, S_{\mathfrak{g} / \mathfrak{h}} \otimes E_{\mu}\right)$ given by the evaluation at the identity. Here the action of $\mathfrak{a}_{\mathfrak{h}}$ on $W_{\lambda_{\mu}} \otimes \mathbf{C}_{-2 \rho_{\mathfrak{h}}}$ is given by $\left.\left(\mu+\rho_{\mathfrak{h}}\right)\right|_{\mathfrak{a}_{\mathfrak{h}}}$, while the action by $N \cap H$ is trivial (see [MZ14b, Section 1.4.4]).

Let $F=F_{\nu}$ be the irreducible finite-dimensional representation of $G$ with highest weight $\nu \in(\mathfrak{a}+\mathfrak{t})^{*}$ such that it is $\Delta^{+}(\mathfrak{h})$-dominant and assume that $\left.\nu\right|_{\mathfrak{a}}=0$. Then, as before, we get 
maps

$$
C^{\infty}\left(G / P, \widetilde{\mathcal{W}}_{\mu}\right) \otimes F_{\nu} \stackrel{\alpha^{P}}{\longrightarrow} C^{\infty}\left(G / P, \widetilde{\mathcal{W}}_{\mu} \otimes \mathcal{F}_{\nu}\right) \stackrel{p_{\mu+\nu}^{P}}{\longrightarrow} C^{\infty}\left(G / P, \widetilde{\mathcal{W}}_{\mu+\nu}\right)
$$

Here $\alpha^{P}$ is defined similarly to $\alpha^{H}$ and $\alpha^{K}$ as in Proposition 2.1. The map $p_{\mu+\nu}^{P}$ is the map induced as before by an $M$-intertwining map (see Section 3)

$$
A_{\mathfrak{b} \cap \mathfrak{m}}^{\infty}\left(\lambda_{\mu}\right) \otimes F_{\nu \mid \mathfrak{t}} \rightarrow A_{\mathfrak{b} \cap \mathfrak{m}}^{\infty}\left(\lambda_{\mu+\nu}\right)
$$

Theorem 4.1. The following diagram is commutative

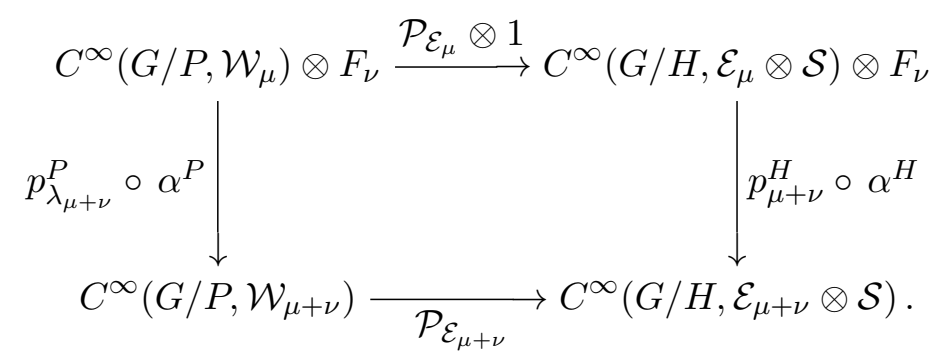

Indeed, for all $g \in G, w \in F_{\nu}$ and $f \in C^{\infty}\left(G / P, \mathcal{W}_{\mu}\right)$, we have :

$$
\begin{aligned}
\left(\mathcal{P}_{\mathcal{E}_{\mu+\nu}} \circ p_{\lambda_{\mu+\nu}}^{P} \circ \alpha^{P}\right)(f \otimes w)(g) & =\int_{H \cap K} \ell \cdot\left[p_{\lambda_{\mu+\nu}}^{P}\left((f(g \ell)) \otimes(g \ell)^{-1} \cdot w\right)(e)\right] d \ell \\
& =\int_{H \cap K} \ell \cdot\left[p_{\lambda_{\mu+\nu}}^{M}\left((f(g \ell)) \otimes(g \ell)^{-1} \cdot w\right)(e)\right] d \ell \\
& =\int_{H \cap K} \ell \cdot\left[p_{\mu+\nu}^{M \cap H}\left((f(g \ell)(e)) \otimes(g \ell)^{-1} \cdot w\right)\right] d \ell
\end{aligned}
$$

by equation (16) .

Recall that $E_{\tilde{\mu}}$ is the irreducible representation of $M \cap H$ with highest weight $\left.\mu\right|_{\mathfrak{t}_{\mathfrak{h}}}$ with respect to $\Delta^{+}(\mathfrak{m} \cap \mathfrak{h})$, and $V_{0}(\mu)$ the $H$-representation defined in Section 3. The following diagram is commutative:

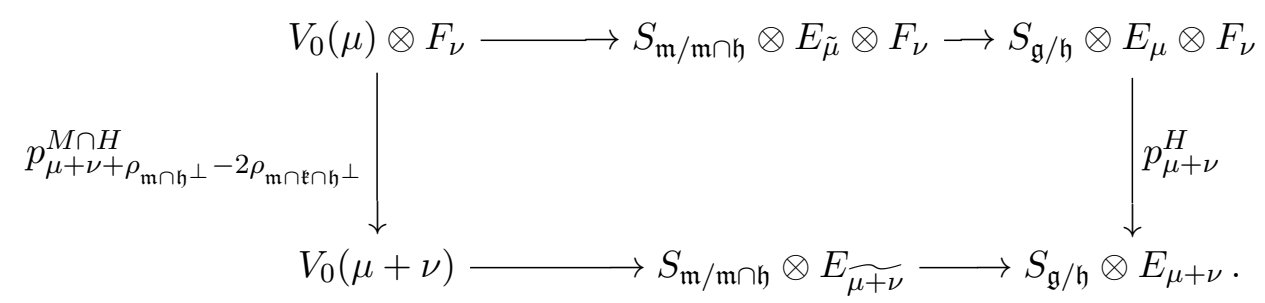


So we get

$$
\begin{aligned}
\left(\mathcal{P}_{\mathcal{E}_{\mu+\nu}} \circ p_{\lambda_{\mu+\nu}}^{P} \circ \alpha^{P}\right)(f \otimes w)(g) & =\int_{H \cap K} \ell \cdot\left[p_{\mu+\nu}^{H}\left((f(g \ell)(e)) \otimes(g \ell)^{-1} \cdot w\right)\right] d \ell \\
& =\int_{H \cap K} p_{\mu+\nu}^{H}\left([\ell \cdot(f(g \ell)(e))] \otimes g^{-1} \cdot w\right) d \ell
\end{aligned}
$$

by $H$-equivariance of $p_{\mu+\nu}^{H}$

$$
=p_{\mu+\nu}^{H}\left(\int_{H \cap K}[\ell \cdot(f(g \ell)(e))] d \ell \otimes g^{-1} \cdot w\right)
$$

by linearity of integration

$$
=p_{\mu+\nu}^{H} \circ \alpha^{H} \circ\left(\mathcal{P}_{\mathcal{E}_{\mu}} \otimes 1\right)(f \otimes w)(g) .
$$

\section{REFERENCES}

[AS77] M. F. Atiyah and W. Schmid. A geometric construction of the discrete series for semisimple Lie groups. Invent. Math., 42:1-62, 1977.

[Kos99] B. Kostant. A cubic Dirac operator and the emergence of Euler number multiplets of representations for equal rank subgroups. Duke Math. J., 100(3):447-501, 1999.

[KR00] A. Korányi and H. M. Reimann. Equivariant first order differential operators on boundaries of symmetric spaces. Invent. Math., 139(2):371-390, 2000.

[KV95] A. W. Knapp and D. A. Vogan, Jr. Cohomological induction and unitary representations, volume 45 of Princeton Mathematical Series. Princeton University Press, Princeton, NJ, 1995.

[KW76] A. Knapp and N. Wallach. Szegö kernels associated with discrete series. Invent. Math, 34:163-200, 1976 .

[Lan00] G. D. Landweber. Harmonic spinors on homogeneous spaces. Represent. Theory, 4:466-473 (electronic), 2000.

[MZ06] S. Mehdi and R. Zierau. Principal series representations and harmonic spinors. Adv. Math., 199(1):128, 2006.

[MZ14a] S. Mehdi and R. Zierau. The Dirac cohomology of a finite dimensional representation. Proc. Amer. Math. Soc., 142(5):1507-1512, 2014.

[MZ14b] S. Mehdi and R. Zierau. Harmonic spinors on reductive homogeneous spaces. In Developments and retrospectives in Lie theory, volume 37 of Dev. Math., pages 161-181. Springer, Cham, 2014.

[Par72] R. Parthasarathy. Dirac operator and the discrete series. Ann. of Math. (2), 96:1-30, 1972.

[Pru10] N. Prudhon. Remarques à propos de l'opérateur de dirac cubique. CRAS, 348(23-24):1249-1252, 2010.

[Wal92] N. R. Wallach. Real reductive groups II, volume 132 of Pure and Applied Mathematics. Academic Press, Boston, 1992.

Université de Lorraine, Institut Elie Cartan de Lorraine, UMR 7502 - CNRS, France

E-mail address: salah.mehdi@univ-lorraine.fr

E-mail address: nicolas.prudhon@univ-lorraine.fr 